\title{
Portfolio size, non-trading frequency and portfolio return autocorrelation
}

\author{
Patricia L. Chelley-Steeley \\ $\&$ \\ James M. Steeley
}

\begin{abstract}
In this paper we re-examine the relationship between non-trading frequency and portfolio return autocorrelation. We show that in portfolios where security specific effects have not been completely diversified, portfolio autocorrelation will not increase monotonically with increasing non-trading, as indicated in Lo and MacKinlay (1990). We show that at high levels of nontrading, portfolio autocorrelation will become a decreasing function of non-trading probability and may take negative values. We find that heterogeneity among the means, variances and betas of the component securities in a portfolio can act to increase the induced autocorrelation, particularly in portfolios containing fewer stocks. Security specific effects remain even when the number of securities in the portfolio is far in excess of that considered necessary to diversify security risk.
\end{abstract}

JEL: G12

Keywords: Portfolio return autocorrelation, non-trading, diversification

Professor Patricia L. Chelley-Steeley, Birmingham Business School, University of Birmingham, Birmingham, B15 2TY, UK. Tel: +44-121-204-3060. Email: p.l.chelley-steeley@aston.ac.uk. Professor James M. Steeley (corresponding author), Aston Business School, Aston University, Birmingham, B4 7ET, UK. Tel: +44-121-204-3248. Email: j.m.steeley@aston.ac.uk. We acknowledge the helpful comments of an anonymous reviewer of this journal. 


\title{
Portfolio size, non-trading frequency and portfolio return autocorrelation
}

\begin{abstract}
In this paper we re-examine the relationship between non-trading frequency and portfolio return autocorrelation. We show that in portfolios where security specific effects have not been completely diversified, portfolio autocorrelation will not increase monotonically with increasing non-trading, as indicated in Lo and MacKinlay (1990). We show that at high levels of non-trading, portfolio autocorrelation will become a decreasing function of non-trading probability and may take negative values. We find that heterogeneity among the means, variances and betas of the component securities in a portfolio can act to increase the induced autocorrelation, particularly in portfolios containing fewer stocks. Security specific effects remain even when the number of securities in the portfolio is far in excess of that considered necessary to diversify security risk.
\end{abstract}




\section{Introduction}

When securities respond nonsynchronously to market-wide news, portfolios comprised of those securities will display autocorrelation in returns. This characteristic of portfolio returns is important to recognize and understand because studies by Scholes and Williams (1977), Dimson (1979) and Cohen et al (1983) showed that this autocorrelation can cause a downward bias in the measurement of market betas. These studies each proposed methods to mitigate the effects of nonsynchronous trading on the estimation of beta.

However, studies by Atchison et al (1985), using the Scholes and Williams (1977) model, and Lo and MacKinlay (1990a), using their generalization of that model, have suggested that only a relatively small proportion of observed autocorrelations can be accounted for by nonsynchronous trading. Atchison et al (1985) predicted a 4 percent daily first order autocorrelation coefficient due to nonsynchronous trading, against an observed level of around 30 percent. Lo and MacKinlay (1990a) reported a weekly first order autocorrelation of 46 percent for observed returns to a portfolio of small firms. However, their model suggested that the autocorrelation induced by the characteristics of observed nonsynchronous trading was less than 9 percent. A generalization of the Lo and MacKinlay (1990a) model by Boudoukh et al (1994), however, increased the predicted first order weekly autocorrelation due to nonsynchronous trading to 18 percent. Kadlec and Patterson (1999), using simulations based on observed trading times, found that over fifty percent of observed daily autocorrelations could be accounted for by thin trading, but around twenty five percent of observed weekly autocorrelation could be accounted for by thin trading. This places their result for weekly returns in between those of Lo and MacKinlay (1990) and Boudoukh et al (1994) results.

A common feature in the models that relate portfolio autocorrelation to nonsynchronous trading is that they use model properties that require the number of securities in a portfolio to have increased without limit. ${ }^{1}$ While the number of stocks required for the diversification of idiosyncratic risk in a stock portfolio is well documented, ${ }^{2}$ the relationship between the number of stocks in a portfolio and the portfolio autocorrelation generated by nonsynchronous trading has not been subject to the

\footnotetext{
1. See, for example, equations (8) and (9) in Atchison et al (1987) and Proposition 2.2 (equation 2.26) in Lo and MacKinlay (1990). Lo and MacKinlay (1990) liken their asymptotic approximation to that used in the Arbitrage Pricing Theory literature, see, for example, Chamberlain (1983) and Chamberlain and Rothschild (1983).

2. See, for example, Statman (1987).
} 
same level of scrutiny. ${ }^{3}$ We show that security means and variances may continue to influence the portfolio return autocorrelation in a portfolio that might be considered well-diversified from the viewpoint of portfolio risk.

The objective of this study is to demonstrate how the portfolio autocorrelation properties of the leading model of nonsynchronous trading, Lo and MacKinlay (1990), change in portfolios that, despite containing a large number of securities, continue to be influenced by security-specific variation. A key property of the Lo and MacKinlay (1990) model is that there is a positive and strictly convex relation between stock trading infrequency and portfolio autocorrelation in portfolios where the number of securities has increased without limit. We show that when portfolios are not sufficiently large for security specific effects to have been eliminated, the relationship between non-trading and portfolio return autocorrelation is no longer strictly convex and always positive. We find that at high levels of non-trading, portfolio return autocorrelation can become a decreasing function of the non-trading probability. Moreover, for portfolios containing a small number of securities, portfolio autocorrelation may take negative values. These potential reductions in the portfolio return autocorrelation predicted by the Lo and MacKinlay model (1990) are more pronounced in portfolios containing a small number of securities. These reductions may be offset if the securities are sufficiently heterogeneous in their return means, variances and covariances.

We estimate the frequency of non-trading for stocks listed on NYSE/AMEX and NASDAQ to show that it remains an important consideration for portfolio autocorrelation. We use these estimates to compute the effects of non-trading on portfolio autocorrelation where the portfolios are influenced by security specific parameters. To maintain consistency with the earlier work of Lo and MacKinlay (1990), Boudoukh et al (1994) and Kadlec and Patterson (1999), we use their market wide parameters together with a range of security specific parameters drawn from widely available historical estimates.

Our results have two important implications. First, empirical studies that adjust risk measures for thin trading by reference to portfolio autocorrelation coefficients, using models such

3. An empirical study by Perry (1985) found that portfolio return autocorrelations increased as the number of securities increased in both a portfolio of small firms and a portfolio of large firms, but he did not explicitly measure the degree of non-trading in each portfolio. Steeley (1997) extended this approach to examine how portfolio return cross autocorrelations and time diversification are influenced by the number of securities in a portfolio. Atchison et al (1987) used the Scholes and Williams (1977) model of non-synchronous trading, but still used asymptotic properties in constructing their predicted autocorrelation function. The original observation of index return autocorrelation is Fisher (1966). 
as those of Scholes and Williams (1977) and Dimson (1979), may have the potential to overcorrect the measures if the predicted autocorrelation is actually quite low. ${ }^{4}$ Second, the observation of high and homogeneous levels of infrequent trading alongside high levels of portfolio return autocorrelation must mean that factors, other than nonsynchronous trading, are generating the observed autocorrelations. ${ }^{5}$

The remainder of the paper is structured as follows. The next section develops the properties of the Lo and MacKinlay (1990) model of nonsynchronous trading when portfolios continue to influenced by security specific parameters. Section 3 presents estimates of the frequency of non-trading among stocks listed on the NYSE/AMEX and NASDAQ, and presents contrasting estimates of portfolio return autocorrelation functions for portfolios with and without the influence of security specific parameters. This is undertaken both in the case where the securities are assumed to be homogeneous and in the case where heterogeneity among the securities is introduced. These estimates identify the relationships between portfolio return autocorrelation and the number of securities in a portfolio, the probability of non-trading and other, return-based, characteristics of the component securities. Section 4 summarizes and concludes.

\section{Implied autocorrelations in portfolios with a finite number of securities}

The first-order autocorrelation of the observed returns, $R_{t}, t=1,2, \ldots, T$, for a portfolio of $i=$ $1,2, \ldots, n$, securities is

$$
\operatorname{Corr}\left(R_{t}, R_{t+1}\right)=\operatorname{Cov}\left(R_{t}, R_{t+1}\right) / \operatorname{Var}\left(R_{t}\right)
$$

where

\footnotetext{
4. Studies by Griffin and Oomen (2012) and Zhang (2012) show that estimated contemporaneous return covariances, and hence estimated betas, can be affected both by nonsynchronous trading and by the existence of i.i.d. microstructure noise associated with high-frequency sampling of realized returns. Both of these papers and an earlier unpublished study by Martens (2004), which uses the Lo and MacKinlay framework, show that there is a trade-off between the downward bias in contemporaneous covariance induced by nonsynchronous trading and the increase in the variance (imprecision) of the covariance estimate arising from microstructure noise, such as bid-ask bounce. None of the methods to adjust covariance measures, Scholes and Williams (1977), Dimson (1979), Cohen et al (1983) and Hayashi and Yoshida (2005), is free from this trade-off. Both Griffin and Oomen (2012) and Zhang (2012) develop optimal sampling frequencies to balance this trade-off, and show that the ranking of the proposed covariance adjustments depends on the scale of the microstructure noise. While both of these papers and our work assume a fixed time interval, a natural extension would be to allow time varying moments of returns. This is an open topic for future research.

5. Portfolio autocorrelations have alternatively been associated with many other factors. For example, Keim and Stambaugh (1986) and Conrad and Kaul (1988) have shown that time varying expected returns can induce portfolio return autocorrelation. Mech (1993) has shown that frictions due to the bid-ask spread can cause return dependence. Sias and Starks (1994) and Brindranath et al (1995) relate changes in institutional holding patterns relate to portfolio return autocorrelation while Boulatov et al (2013) examines how order flow patterns of informed traders influence portfolio serial correlation. Anderson et al (2011) provides a review of the alternative explanations for portfolio return autocorrelation.
} 


$$
R_{t}=\sum_{i=1}^{n} \omega_{i} R_{i, t}, \quad \text { subject to } \sum_{i=1}^{n} \omega_{i}=1
$$

and where $R_{i, t}$ is the return on security $i$ at time $t$, and $\omega_{i}$ is the weight on security $i$ in the portfolio. For an equally-weighted portfolio, we obtain on substituting (2) into (1),

$$
\operatorname{Corr}\left(R_{t}, R_{t+1}\right)=\frac{\sum_{i=1}^{n} \operatorname{Cov}\left(R_{i, t}, R_{i, t+1}\right)+\sum_{i=1}^{n} \sum_{j=1, \neq i}^{n} \operatorname{Cov}\left(R_{i, t}, R_{j, t+1}\right)}{\sum_{i=1}^{n} \operatorname{Var}\left(R_{i, t}\right)+\sum_{i=1}^{n} \sum_{j=1, \neq i}^{n} \operatorname{Cov}\left(R_{i, t}, R_{j, t}\right)}
$$

which expresses the portfolio return autocorrelation in terms of the autocovariances, crossautocovariances, covariances and variances of the returns of the component securities.

\subsection{The Lo and MacKinlay (1990) model of nonsynchronous trading}

The models of Scholes and Williams (1977) and Lo and MacKinlay (1990) establish the relationship between the terms in equation (3) and the variance-covariance matrix of an underlying unobservable returns process, when securities trade nonsynchronously. Specifically, Lo and MacKinlay (1990) assume that unobservable security returns, $R_{i, t}^{*}$ are determined by the stochastic model,

$$
R_{i, t}^{*}=\mu_{i}+\beta_{i} \Lambda_{t}+\varepsilon_{i, t}
$$

where $\Lambda_{t}$ is a zero-mean common factor, with variance $\sigma_{\Lambda}^{2}, \mu_{i}$ is the expected unobserved return to security $i, \beta_{i}$ is the beta of security $i$ with the common factor and $\varepsilon_{i, t}$ are cross-sectionally and temporally independent idiosyncratic shocks. They further assume that the common factor $\Lambda_{t}$ is independently distributed and independent of $\varepsilon_{i, t-s}$ for all $i, t$ and $s$.

In each period $t$, security $i$ is assumed not to trade with a probability $p_{i}$. If the security does not trade, its observed return for period $t$ is zero, while its unobservable return is given by equation (4) above. If the security does trade, its observed return is the sum of the unobserved returns for that period and all past consecutive periods for which it did not trade. This structure causes movements in the common factor to be reflected in security prices only when securities trade. 
For this model, Lo and MacKinlay (1990, Proposition 2.1) establish the first and second moment properties of observed security returns as a function of the non-trading probabilities and the parameters of the unobserved returns in equation (4), specifically,

$$
\begin{aligned}
& \operatorname{Cov}\left(R_{i, t}, R_{i, t+s}\right)= \begin{cases}\sigma_{i}^{2}+\frac{2 p_{i}}{\left(1-p_{i}\right)} \mu_{i}^{2} & \text { for } s=0 \\
-\mu_{i}^{2} p_{i} & \text { for } s=1\end{cases} \\
& \operatorname{Cov}\left(R_{i, t}, R_{j, t+s}\right)= \begin{cases}\frac{\left(1-p_{i}\right)\left(1-p_{j}\right)}{\left(1-p_{i} p_{j}\right)} \beta_{i} \beta_{j} \sigma_{\Lambda}^{2} & \text { for } s=0 \\
\frac{\left(1-p_{i}\right)\left(1-p_{j}\right)}{\left(1-p_{i} p_{j}\right)} p_{j} \beta_{i} \beta_{j} \sigma_{\Lambda}^{2} & \text { for } s=1\end{cases}
\end{aligned}
$$

where $\operatorname{Var}\left(R_{i, t}^{*}\right)=\sigma_{i}^{2}$ and $p_{i}<1, \forall i .^{6}$

Substituting (5) and (6) into equation (3), expresses the portfolio return autocorrelation function in terms of the non-trading probabilities and the parameters of the unobserved returns in equation (4). For a representative beta and non-trading probability for all securities in the portfolio, Lo and MacKinlay (1990) show that, as the number of securities in the portfolio increases without limit, the first order portfolio return autocorrelation is (asymptotically equal to) $p$ where $p=p_{i} \forall i$. Intuitively, their proof of this result runs as follows. As the number of securities is increased without limit, so the terms in (5) approach zero, and so (3) is approximately the ratio of the terms in (6). These terms immediately divide through to leave $p_{j}=p$ the representative probability. In the case of autocorrelation of higher orders, they show that the portfolio return autocorrelation is equal to the power function $p^{s}$, where $s$ is the autocorrelation order (lag).

\subsection{Implications for finite-sized portfolios}

If the number of securities does not increase without limit (but still maintaining a representative security assumption), we show that the portfolio return autocorrelation function is no longer a

5. The results in (5) and (6), and those derived from here onwards, are not defined for $p_{i}=1$, the case of complete non-trading, when, at least intuitively, all covariances and variances will be zero. 
power function of non-trading probability. Specifically, in the appendix we derive the following result.

Proposition 1. Under the definition of unobservable security returns in equation (4), using the variance, covariance and autocovariance properties set out in equations (5) and (6) and making a representative security assumption, the first order return autocorrelation of a portfolio containing n securities, where $n$ has not increased without limit, is

$$
\operatorname{Corr}\left(R_{t}, R_{t+1}\right)=\frac{(1-p) p}{\theta(1+p)-2 p}
$$

where

$$
\theta=\frac{(n-1)(p-1) \beta^{2} \sigma_{\Lambda}^{2}+\sigma^{2}(1-p)}{(n-1)(p-1) \beta^{2} \sigma_{\Lambda}^{2}-\mu^{2}(1+p)}
$$

Our Proposition 1 introduces the term $\theta$, which we identify to be the adjustment to the portfolio return autocorrelation that is required for portfolios with a finite number of securities. This term shows that in the case of portfolios containing a finite number of securities, portfolio return autocorrelation may continue to be influenced by the (representative) beta of the securities, the risk of the common factor, $\sigma_{\Lambda}^{2}$, the (representative) mean and variance of the securities, $\mu, \sigma^{2}$, respectively, and the number of securities in the portfolio, $n$, through the adjustment term $\theta{ }^{7}$

The earlier result of Lo and MacKinlay (1990, Proposition 2.2, equation 2.26) that portfolio return autocorrelation is a linear function of non-trading probability in the case of portfolios where the number of securities has increased without limit follows as a special case of our Proposition 1. In fact, any combination of security-level parameter values and the number of securities that leads to $\theta=1$, will cause equations (7) and (8) to simplify immediately to $\operatorname{Corr}\left(R_{t}, R_{t+1}\right)=p$. One such possibility is when $n \rightarrow \infty$ and so $\theta \rightarrow 1$, which will then confirm the asymptotic result of Lo and MacKinlay (1990) that they obtained through other means. ${ }^{8}$

The dependence of portfolio return autocorrelation on the number of component securities, as shown in the adjustment term, equation (8), means in developing stock markets where indices

7. In Lo and MacKinlay (1990), the representative non-trading probability and beta are the asymptotic sample means. For the finite sample case that we have developed, an explicit representative security assumption is needed. While this is an essential base case for examination, various dimensions of this assumption are relaxed later in the paper.

8. While the focus here is on the first-order autocorrelation coefficient of portfolio returns, as these have been found to be by far the most economically and statistically significant statistics in empirical work, the changes to the other second moments results in Lo and MacKinlay (1990, proposition 2.1) when $n$ has not increased without limit can be extracted easily from the derivations in the appendix. 
may be being constructed from only a very small number of securities that the return autocorrelations in these indices are likely to be heavily dependent on security level means, variances and covariances. The adjustment term provides a route to understand how these security level effects are contributing to index autocorrelation.

\subsection{Time aggregation}

In this section, we examine the properties of the extended version of the Lo and MacKinlay (1990) model that contrasts the autocorrelation of portfolio returns sampled at different intervals. ${ }^{9}$ For example, the properties of weekly or monthly observed returns can be written in terms of, say, daily non-trading probabilities.7

Denoting $R_{\tau}(q)$ as the observed return on a portfolio at time $\tau$, where one unit of $\tau$ time is equal to $q$ units of $t$ time, such that

$$
R_{\tau}(q)=\sum_{t=(\tau-1) q+1}^{\tau q} R_{t}
$$

Lo and MacKinlay (1990, Proposition 3.2, equation 3.11) show that under their asymptotic assumption, the first order autocorrelation function of the temporally aggregated returns is

$$
\operatorname{Corr}\left(R_{\tau}(q), R_{\tau+1}(q)\right)=\frac{\left(1-p^{q}\right)^{2} p}{q\left(1-p^{2}\right)-2 p\left(1-p^{q}\right)}
$$

Equation (10) shows that the effect of temporal aggregation on (calendar independent) observed portfolio returns is to induce a strict convexity into the relation between autocorrelation and non-trading probability, $p<1$. This can be seen in Figure 1, and replicates the figure in panel (d) of Figure 1 of Lo and MacKinlay (1990, p.196). This plot also shows that the degree of convexity increases with the coarseness of the observed return sampling interval.

[Figure 1]

9. Later studies, such as Boudoukh et al (1994) and Kadlec and Patterson (1999) also adopted this approach. 
In the appendix, we derive the companion result for the case where the number of securities in the portfolio has not increased without bound. Specifically, we obtain

Proposition 2. Under the definition of unobservable security returns in equation (4), using the variance, covariance and autocovariance properties set out in equations (5) and (6) and making a representative security assumption, the first order autocorrelation coefficient of time-aggregated returns in a portfolio of $n$ securities, where $n$ has not increased without limit, is

$$
\operatorname{Corr}\left(R_{\tau}(q), R_{\tau+1}(q)\right)=\frac{\left(1-p^{q}\right)^{2} p}{\theta q\left(1-p^{2}\right)-2 p\left(1-p^{q}\right)}
$$

Where $\theta$ is as defined in (8).

Our result, equation (11), generalizes the relation in equation (10) for the case where the number of securities in the portfolio has not increased without bound. Equation (11) differs only by the inclusion of $\theta$ from the result of Lo and MacKinlay (1990), shown in equation (10). Thus, the adjustment to the portfolio autocorrelation predicted in the case of portfolios with a finite number of component securities does not depend on the degree of temporal aggregation of the portfolio returns. Only in circumstances where the security-level parameters within $\theta$ give rise to $\theta=1$, will our generalized autocorrelation relation be the same as that derived by Lo and MacKinlay and display the convex and increasing pattern seen in Figure 1 . In circumstances where $\theta \neq 1$, the return autocorrelation of portfolios containing a finite number of securities may no longer conform to these patterns.

In the next section, we investigate this issue by estimating the portfolio return autocorrelation coefficients, using equations (8) and (11), for varying mixtures of portfolio size, non-trading probability, and representative security characteristics, using parameters calibrated to market data.

\section{Calibration Analysis}

In this section, to demonstrate the economic relevance of our adjustment term for the calculation of the effects of nonsynchronous trading on portfolio return autocorrelation, we undertake a 
calibration analysis. In this analysis, we show how the autocorrelation estimates generated by the model of Lo and MacKinlay (1990) change when portfolios contain a finite number of securities rather than the number of securities having increased without bound. We re-examine both the original results of Lo and MacKinlay (1990) and the extension of this work by Boudoukh et al (1994).

Our generalization of the portfolio autocorrelation function contains the adjustment term, $\theta$. This depends not only upon the representative probability of non-trading, $p$, but also upon security level parameters and the number of securities in the portfolio. Specifically, $\theta$ depends upon $\beta$, the (representative) beta of the securities, the risk of the common factor, $\sigma_{\Lambda}^{2}$, the (representative) mean and variance of the securities, $\mu, \sigma^{2}$, and $n$, the number of securities in the portfolio. To examine the differences between our generalized autocorrelation function and the special case considered Lo and MacKinlay (1990), it is necessary to provide values for each of these parameters. Below we describe how we set the values of these parameters.

From equation (4), the variance of the returns of a representative security, $\sigma^{2}$, can be decomposed as 9

$$
\sigma^{2}=\beta^{2} \sigma_{\Lambda}^{2}+\operatorname{var}(\varepsilon)
$$

By assuming that the representative idiosyncratic return variance deviation, $\operatorname{var}(\varepsilon)$ is proportional to the variance of the common factor, the overall variance of returns, $\operatorname{var}(\varepsilon)=\zeta \sigma_{\Lambda}^{2}$, equation (12), becomes

$$
\begin{aligned}
& \sigma^{2}=\left(\beta^{2}+\zeta\right) \sigma_{\Lambda}^{2} \\
& \sigma^{2}=\phi \sigma_{\Lambda}^{2}
\end{aligned}
$$

where the variance ratio $\phi=\left(\beta^{2}+\zeta\right)=\sigma^{2} / \sigma_{\Lambda}^{2}$ measures the variance of security relative to the variance of the common factor. If the representative security mean return is set relative to the standard deviation of returns using the reward ratio, $\xi=\mu / \sigma$, then the adjustment term, $\theta$, can be written as follows

$$
\theta=\frac{(n-1)(p-1) \beta^{2}+\phi(1-p)}{(n-1)(p-1) \beta^{2}-\xi^{2} \phi(1+p)}
$$

Reconfiguring $\theta$ in this way removes the variance of the common factor, and means that only the reward ratio, $\xi$, the variance ratio, $\phi$ and beta need to be assigned values. 
We set the bench-mark value for $\xi$ by reference to historical estimates of annualized means and standard deviations of stock returns, such as those produced by Dimson et al (2001). These report annualized mean (excess) returns of around 8 percent, standard deviations of around 20 percent, and a reward ratio of around 0.41 for US stocks. Thus, we use $\xi=0.41$ as the bench-mark value for the reward ratio. We set the bench-mark value for $\phi$ to maintain consistency with the parameterization of the Lo and MacKinlay (1990) model by Kadlec and Patterson (1999) and also the historical estimates used to parameterize the reward ratio. Kadlec and Patterson (1999) use a value for the variance of the common factor that implies a value in the region of $\phi=4$, when beta is set at unity, and so we take this as our base case. ${ }^{10}$

To maintain consistency with Lo and MacKinlay (1990), we use hourly non-trading probabilities in our calculations and then express the induced autocorrelations as a function of daily non-trading probabilities. Thus, for weekly observed returns, we aggregate hourly returns having a non-trading probability of, say, 80.4 percent over 30 periods (6 hours per day), rather than aggregating daily returns having a non-trading probability of 27 percent $\left(.804^{6}=0.27\right)$ over 5 periods (days). We convert all the other parameter values to hourly equivalents also.

\subsection{Homogeneous non-trading probabilities and betas}

To explore the properties of our generalized autocorrelation function, equations (11) and (14), we plot the estimated value of the function for varying non-trading probabilities and portfolio sizes, holding fixed the security level parameters. In Figure 2, the generalized autocorrelation function is estimated using a beta of one, a variance ratio of $\phi=4$ and a reward ratio, $\xi=0.41$, for portfolio returns of weekly, monthly, quarterly and annual frequencies. At the rear of each of the four graphs are the values of the function in the case where the number of securities has increased without limit, and which (as previously shown by Lo and MacKinlay (1990),increases towards unity. Each of these cases, which are for portfolios where the number of securities has increased without bound, support the empirical findings of both Perry (1985) and Atchison et al (1987) that portfolio return autocorrelation is an increasing function of the number of securities.

\section{[Figure 2]}

10. We use a unit beta as our base case to enable direct comparison of our results to those in the homogeneous beta case examined in Boudoukh et al (1994). Variable security betas are considered in Section 3.2 below that re-examines the heterogeneous portfolios considered by Boudoukh et al (1994). 
By contrast to these cases, the portions of the five graphs for portfolios containing finite numbers of securities, show that the autocorrelation functions are no longer always an increasing function of non-trading probability. Instead, they are a decreasing function after some relatively high non-trading probability. Although difficult to detect visually from Figure 2, the autocorrelation functions briefly take small negative values at some non-trading probability levels in excess of 99 percent. Moreover, the graphs also show that the non-trading probability level at which the autocorrelation function begins to decrease is itself an increasing function of the number of securities in the portfolio.

Table 1 shows some estimated values of the generalized autocorrelation function for portfolio returns recorded at weekly, monthly, quarterly and annual intervals, for portfolios comprising 10, 20, 100 and 500 securities, for daily non-trading probabilities of 27, 60, 80 and 95 percent. The non-trading probability of 27 percent corresponds to the observation by Boudoukh et al (1994) that the average non-trading probability for the smallest decile of US stocks is around 27 percent. ${ }^{11}$ This observation received subsequent corroboration in the work of Lesmond, Ogden and Trzcinka (1999), who found that the proportion of zero volume days in the small firm decile for NYSE/AMEX stocks in the CRSP database is 27.81 percent, in the three-year period 1988-1990. In Figure 3, we update these findings and provide a series of annual measures of the non-trading among small firms in both the NYSE/AMEX and NASDAQ markets. It can be seen that while the proportion of zero volume days has declined since the early Nineties, there have been three occasions when it has risen once again, including in 2008. Across the entire sample, the average percentage of non-trading days is around 23 percent across the two markets, but this hides extensive historic variation that will be important to account for in research that covers these time periods. For example, in 1992, the percentage of zero volume days on NASDAQ small firm stocks was 46 percent. The even higher non-trading probabilities used as example levels in Table 1 not only recognize that for other less developed markets the probabilities of non-trading among small stocks may be even higher, but also help to reveal the properties of the generalized autocorrelation function.

[Figure 3]

11. Using evidence in Foerster and Keim (1993) and Keim (1989). 
The portfolio return autocorrelation values in Table 1 are obtained for nine different combinations of the variance ratio, $\phi=2,4,9$, and the reward ratio, $\xi=0.19,0.41,0.65$, where the middle case in each of the triples is the bench-mark value used in Figure 2. The upper and lower variance ratio values represent a range of annualized standard deviations of security returns of between 15 percent and 32 percent, the latter being consistent with estimates of the standard deviation of the returns of small capitalization stocks reported in sources such as Dimson et al (2001). The reward ratios, combined with these variance ratios represent a range of annualized excess returns of between 3 percent and 20 percent.

[Table 1]

In Table 1 , the rows marked $n \rightarrow \infty$ are the autocorrelations predicted by the model of Lo and MacKinlay (1990) for the case where the number of securities in the portfolio has increased without limit. They do not depend on the security level parameters and so are constant across the row in the table. They are specific examples of points on the curves shown in Figure 1.

Where the number of securities in the portfolio has not increased without bound, the value of the autocorrelations are no longer independent of the security level parameters or the number of securities in the portfolio. This can be seen clearly in the variation in the autocorrelation coefficients in the other rows of Table 1, which are for portfolios containing, as examples, 10, 20, 100 or 500 securities. The column labelled $\phi=4$ and $\xi=0.41$ is used as a bench-mark case from which the effects of different security level parameters and numbers of securities can be gauged. This column corresponds to a portfolio having stocks with mean excess annual stock returns of 8 percent and an annualized standard deviation of returns of 20 percent. The values in the rows of this central column of the table are specific examples of points in the four graphs in Figure 2. The remaining columns of Table 1, which consider changed values of these parameters show, therefore, how these graphs in Figure 2 may vary when the security level parameters are different from this bench-mark case. Columns with greater values of $\phi$ represent an increase in the representative idiosyncratic volatility of the component stocks in the portfolio, while columns with increased values of $\xi$ represent an increase the representative reward to risk ratio of the securities in the portfolio. Decreases in these parameter values represent corresponding decreases in idiosyncratic risk and reward ratio, respectively. 
For large portfolios, of 500 companies, the inclusion of security level information leads to no more than a 1 percentage point reduction in portfolio return autocorrelations from the asymptotic value, in the column headed $n \rightarrow \infty$, for daily non-trading probabilities of 27 and 60 percent. The effects are of similar magnitude at the 80 percent probability level, except for weekly returns where a 2 percentage point reduction is observed, but only when the variance ratio and reward ratios take high values. At the 95 percent probability level, the autocorrelations are reduced less than one percentage point for quarterly or annual returns. For monthly returns, the reduction is limited to around 2 percentage points, and this is only observed for high values of the variance ratio and the reward ratio. For weekly returns, the reductions in autocorrelation coefficients can be greater. For low values of the variance ratio, the impact can amount to a 3 percentage point reduction, while for the high variance ratio, the autocorrelation coefficients can reduce by 12 percentage points (from 85 percent to 73 percent).

For portfolios containing 100 component securities, the effects on portfolio return autocorrelation coefficients of including security level parameters are similar to those observed for portfolios of 500 securities, at non-trading probabilities of 27 percent and 60 percent. At higher levels of non-trading, we observe greater percentage point changes in the portfolio return autocorrelation coefficients than were observed for portfolios of 500 securities. Specifically, for monthly returns, the coefficient can fall by up to 10 percentage points, while for daily returns it can fall by up to 38 percentage points. The changes in the autocorrelation coefficient are larger in the cases where the variance ratio takes on a high value, meaning that the security level parameters matter most when they take relatively large values. For the case of quarterly and annual returns, where it can be seen that the autocorrelations in any case take very low values except in circumstances of extreme thin trading, the effects of the number of securities in the portfolio are more modest, with differences of less than 2 percentage points obtained.

For the portfolios containing 20 securities, which might be regarded as well-diversified from the point of view of the influence of idiosyncratic variance, the generalized portfolio return autocorrelation coefficients are much more noticeably different from the asymptotic values, even when the non-trading probability is 27 percent. Specifically, weekly autocorrelation coefficients will see a reduction from the asymptotic level of 8.9 percent to 5.7 percent, when the variance ratio is high. At the 60 percent non-trading level, the weekly coefficients can reduce to around one half of their asymptotic levels, a drop of around 12 percentage points. At the 80 percent probability 
level, the autocorrelations for annual and quarterly observed returns are affected by around only 2 percentage points, but in the high variance ratio case, monthly return autocorrelations can be reduced by up to 5 percentage points. For weekly returns, the asymptotic autocorrelation of 50 percent can be reduced by 10 percentage points in the low variance ratio scenario, but by 28 percentage points (around three-fifths of its value) in the high variance ratio case. At the 95 percent probability level, autocorrelations for all the return horizons are affected noticeably. For annual returns and quarterly returns, the asymptotic autocorrelations of 4 and 19 percent, respectively, can be reduced by 2 and 8 percentage points. For monthly and weekly returns, the asymptotic autocorrelations of 50 and 84 percent can be reduced to 22 percent and 16 percent, respectively, in the high variance ratio scenario.

For portfolios of just 10 securities, we would expect security idiosyncratic elements to even more dramatically influence portfolio return autocorrelation. ${ }^{12}$ The autocorrelation coefficients in this case are affected in a similar way to those observed for portfolios of 20 securities, but the effects are much more pronounced. For annual returns, the value of the portfolio return autocorrelation coefficients can be halved by including security level parameters, although as these coefficients take relatively small values to begin with, the percentage point reductions are small. For quarterly returns, the effects are similar, except when non-trading probabilities are at 95 percent, when the autocorrelation coefficient can reduce from the asymptotic value of 19 percent to 7 percent. For monthly returns, it is again when non-trading probabilities are extreme that most impact is seen. At the 95 percent non-trading probability, the autocorrelation coefficient can reduce from 50 percent to 13 percent. In the case of weekly returns, even for relatively small return variances and reward ratios, the reduction in the autocorrelation coefficient can be 55 percentage points in the 95 percent non-trading probability case, and 6 percentage points at non-trading probabilities of 60 percent. But, when the reward ratio and the variance ratio take on high values, the autocorrelation coefficients, at a non-trading probability level of 27 percent can reduce to one half of their asymptotic value. When non-trading probabilities are as high as 95 percent, weekly portfolio return autocorrelations can reduce from 84 percent to 8 percent. Even when the reward ratio and the variance ratio take low values, the reduction would be from 84 percent to 30 percent in this case.

12. The number of securities required to diversify a portfolio is usually estimated to be about 15 , see for example, Statman (1987). 
We consider next whether these potential downward adjustment to the asymptotic autocorrelation coefficients, which are found in portfolios containing a finite number of securities, are magnified or mollified when the securities in the portfolio have diverse non-trading, variance ratios and reward ratios.

\subsection{Heterogeneous non-trading probabilities and betas}

Boudoukh et al (1994) demonstrated the importance of considering the influence on non-trading induced portfolio autocorrelation of heterogeneity among the component securities in the portfolio. ${ }^{13}$ They found that the asymptotic weekly portfolio return autocorrelation arising under homogeneity of both beta and non-trading probability of 8.90 percent, the top row in Table 1, increases to 17.82 percent when the beta and non-trading probabilities are both at their most heterogeneous. In Table 1, we have shown that consideration of security level parameters that have not been diversified away in a portfolio containing a fixed number of securities, can reduce the autocorrelation coefficients. In this section, we consider whether these reductions are maintained in portfolios containing more heterogeneous securities. Specifically, we will explore whether the differences between the autocorrelation coefficients in portfolios of unlimited size and those of a finite size are increased or decreased once the homogeneity restriction is relaxed, and how sensitive this difference is to the level of heterogeneity.

To be able to directly compare our results with those of Boudoukh et al (1994), we start with the same four different distributions of non-trading and three distributions of security beta examined in their study. These distributions are imposed across six sub-portfolios, which comprise fractions 5, 20, 25, 25, 20 and 5 percent of stocks in the overall portfolio. Our smallest portfolio, therefore contains 20 securities, such that 1 security represents 5 percent of the portfolio. The nontrading distributions used by Boudoukh et al (1994) were chosen to reflect the characteristics of security trading uncovered by Foerster and Keim (1993). The first distribution is homogeneous, with daily non-trading probabilities of $(27,27,27,27,27,27)$ percent. ${ }^{14}$ The other three distributions are heterogeneous with daily non-trading probabilities $(0,11,21,32,44,55)$ percent, $(0,0,11,32,60,85)$ percent, and $(0,0,0,43,60,85)$ percent. Taking the first heterogeneous case as an

13. Boudoukh et al (1994, Figure 2. p.559) actually conjectured that extreme heterogeneity in non-trading probability could generate decreasing portfolio return autocorrelation. In the previous section, we have shown that decreasing portfolio autocorrelation can also arise with homogeneous non-trading probabilities.

13.Our own calculations of the non-trading probabilities among the stocks in the small firm deciles for both NYSE/AMEX and NASDAQ markets indicate that the non-trading probability has since declined to an average of 23 percent. This can be seen in Figure 3. 
example, this would mean that 5 percent of the portfolio has stocks that always trade, 20 percent of the stocks has an 11 percent probability of non-trading, 25 percent of the stocks has a 21 percent probability of non-trading, a further 25 percent of the stocks has a 32 percent probability of nontrading, a further 20 percent of the stocks has a 44 percent probability of non-trading, while the final 5 percent of stocks has a 55 percent probability of non-trading. The average non-trading probability in each of the four non-trading distributions is 27 percent, but the more heterogeneous the distribution, the higher the variance of non-trading probabilities. The three beta distributions are $(1,1,1,1,1,1),(0.8,0.9,1.0,1.0,1.1,1.2)$, and $(0.8,1.2,1.6,2.0,2.4,2.8)$, and apply across the subportfolios in a similar fashion to the variable non-trading probabilities. The first two distributions of beta have the same mean, while the most heterogeneous distribution has both a higher mean (1.8) and a higher variance. The beta distributions are also those used by Boudoukh et al (1994).

To examine the difference between the asymptotic autocorrelation levels and those obtained using the finite sample formula, we recalculate the weekly autocorrelations using equations (11) and (14). We use the same security level parameters as examined in the case of homogeneity, in Table 1, and consider portfolios containing 20, 100 and 500 securities. As both $\beta^{2}$ and $\zeta$, the ratio of idiosyncratic variance, $\operatorname{var}(\varepsilon)$, to the common factor variance, $\sigma_{\Lambda}^{2}$, have similar multiplicative effects on the total variance of a security, equation (13), we hold $\zeta$ constant at the central scenario value of $\zeta=3$ used in Table 1 that, for a unit beta, implies a variance ratio of $\phi=4$ and an annualized security return standard deviation of around 20 percent. Hence, in the presence of heterogeneous betas, the variance ratio, $\phi$, now takes the value $\phi=\beta^{2}+3$, where the beta values are from the distributions introduced previously. ${ }^{15}$ As the securities in the portfolio are now permitted to have different betas, so the variance ratios of the securities will also be heterogeneous. For any given security return variance, we use the same three scenarios for the reward ratio of mean to standard deviation, $(0.19,0.41,0.65)$, as in homogeneous beta case. For a given scenario, all securities in the portfolio will have the same reward ratio, but as the variances are heterogeneous, then so also will be the security mean returns. Therefore, beta heterogeneity induces heterogeneity in the variances and means of the component securities of the portfolio, but does so in a manner in which the influence of beta heterogeneity can be isolated.

15. This helps isolate the influence of beta heterogeneity in the comparison of autocorrelation coefficients. The case where both beta and idiosyncratic return variance could change between scenarios in the calculation of total security variance was also examined, but the quantitative differences to the results were small and the qualitative conclusions unchanged. 
The autocorrelation coefficients, for heterogeneous betas and non-trading probabilities, are shown in panel (a) of Table 2. As observed by Boudoukh et al (1994), heterogeneity in both beta and non-trading probability acts to increase the measured autocorrelations. Our results, which extend these findings to portfolios of different finite numbers of component securities display some more subtle characteristics. For any given distribution of non-trading probabilities among the component securities in the portfolio, the proportional increase in portfolio return autocorrelation observed as the betas of the securities also become more heterogeneous is larger for portfolios containing smaller numbers of securities. By contrast, for any given distribution of betas among the component securities of the portfolio, the proportional increase in portfolio return autocorrelation observed as non-trading becomes more heterogeneous within the portfolio is roughly equivalent for large and small portfolios. This suggests that the increases in portfolio return autocorrelation are being influenced more by the heterogeneity in security beta than by the heterogeneity in the security non-trading probabilities. It can also be seen that, for any given distribution of heterogeneous betas and non-trading probability, increases in the reward ratio of the component securities now cause barely detectable falls in the autocorrelation coefficients.

[Table 2]

We can alternatively view the same results in Table 2, panel (a) in terms of the influence of the number of securities in a portfolio, given the level of heterogeneity of beta and non-trading probability among the component securities. For any given combination of non-trading probability and beta heterogeneity, the portfolio return autocorrelation coefficient reduces as the portfolio contains a smaller number of component securities. The difference between the influence of nontrading probability heterogeneity and beta heterogeneity also reappears when the results are viewed in these terms. For a given distribution of security betas, the reduction in portfolio autocorrelation coefficient between large and small portfolios is of proportionately equivalent magnitude regardless of the heterogeneity of non-trading. For example, for the beta distribution $(0.8,0.9,1.0,1.0,1.1,1.2)$, the autocorrelation coefficient for a portfolio of 20 securities is around 80 percent of the value of the autocorrelation coefficient for an asymptotically large portfolio, whether the distribution of non-trading probabilities is $(27,27,27,27,27,27)$ or $(0,0,0,43,60,85)$. In the former case, the autocorrelation coefficient reduces from 0.089 to 0.073 , while in the latter case, it reduces from 0.1508 to 0.1193 . By contrast, for any given distribution of non-trading probabilities, the magnitude of the fall in the value of the autocorrelation coefficient as the heterogeneity of beta increases, appears to decrease. However, it only does so when both the mean and the variance of 
the distribution of betas increase. Thus, the 20 percent reductions in the autocorrelation coefficients between the asymptotically large portfolio and the portfolio of 20 securities, seen in the case of the beta distributions of $(1,1,1,1,1,1)$ and $(0.8,0.9,1.0,1.0,1.1,1.2)$, decreases to an 12 percent reduction in the case of the $(0.8,1.2,1.6,2.0,2.4,2.8)$ beta distribution. For example, for the distribution of non-trading probabilities $(0,0,0,43,60,85)$, the autocorrelation coefficient reduces from 0.1397 to 0.1114 in the case of the $(1,1,1,1,1,1)$ beta distribution, from 0.1508 to 0.1193 in the case of the $(0.8,0.9,1.0,1.0,1.1,1.2)$ beta distribution, but from 0.1782 to only 0.1562 in the case of the $(0.8,1.2,1.6,2.0,2.4,2.8)$ beta distribution. So, as was observed when isolating the impacts of heterogeneity, for a given number of securities in a portfolio, heterogeneity in beta seems to exert more influence on portfolio autocorrelations than does heterogeneity in nonsynchronous trading probability. However, it does so in particular when the average beta increases.

Taking the combined impacts of heterogeneity in betas and non-trading probabilities and reduction in portfolio size together, it can be seen that the impact on portfolio return autocorrelation of heterogeneity is considerably larger than (close to double) the impact of reducing the number of securities in a portfolio. Thus, estimating the influence of non-synchronous trading on portfolio return autocorrelation using an asymptotic approximation, which effectively assumes homogeneous betas and non-trading probabilities, would actually become more accurate an approximation for a portfolio of finite size, the more heterogeneous are the component securities.

To determine whether these results are sensitive to the average level of non-trading, we repeat the analysis of panel (a) in Table 2, using non-trading probabilities centred on 60 percent, one of the higher average non-trading levels considered in Table 1. Again each distribution of nontrading probabilities has the same mean, but greater heterogeneity within a distribution results in a higher variance for that distribution. The results of the analysis for the cases of 60 percent average non-trading probabilities are contained in panel (b) of Table $2 .{ }^{16}$ As with the lower average nontrading probabilities, heterogeneity in either or both non-trading probability and beta serves to increase the portfolio return correlation, and, for any given distribution of non-trading probabilities among the component securities in the portfolio, the proportional increase in portfolio return

16. Given the ranges of the heterogeneous non-trading probability distributions centred on a 27 percent probability, it is not possible to match the variances of these distributions when the mean is shifted to 60 percent, without affecting the shape of the distribution. Hence, although the variances increase, as the distributions centred on 60 percent increase in the heterogeneity of the non-trading probabilities of the component securities, the variances are each smaller than in the counterpart distribution centred on 27 percent. 
autocorrelation observed, as the betas of the securities also become more heterogeneous, is larger for portfolios containing smaller numbers of securities. But, in a difference to what was observed for the distributions of lower average non-trading probabilities, for any given distribution of betas among the component securities of the portfolio, the proportional increase in portfolio return autocorrelation observed as non-trading becomes more heterogeneous within the portfolio is smaller for the smaller portfolios. For example, in the case of the $(0.8,0.9,1.0,1.0,1.1,1.2)$ beta distribution, the autocorrelation coefficient for a portfolio of 20 securities increases by 22 percent from 0.1945 , for the $(60,60,60,60,60,60)$ distribution of nonsynchronous trading probabilities to 0.2377 for the $(24,29,44,80,80,80)$ distribution of nonsynchronous trading probabilities. Whereas, for a portfolio of 500 securities, it increases by 29 percent from 0.2569 , for the $(60,60,60,60,60,60)$ distribution of nonsynchronous trading probabilities to 0.3275 for the $(24,29,44,80,80,80)$ distribution of nonsynchronous trading probabilities. This suggests that the influence of nontrading heterogeneity on portfolio return autocorrelation is relatively more important at higher average levels of non-trading. The influence of changes in the reward ratio of the component securities is once again negligible in comparison to other factors.

The contrast between the results for low and high average levels of non-trading also arises when the results are expressed in terms of the number of securities in the portfolio. For any given combination of heterogeneous non-trading probability and beta heterogeneity, the portfolio return autocorrelation coefficient declines as the portfolio contains a smaller number of component securities, as it has done in all prior scenarios. By contrast to the results for the lower level of average non-trading, for a given distribution of security betas, the reduction in portfolio autocorrelation coefficient between large and small portfolios is proportionately larger for portfolios containing more heterogeneous nonsynchronous trading probabilities. For example, in the case of the $(0.8,0.9,1.0,1.0,1.1,1.2)$ beta distribution, the autocorrelation coefficient, for the $(60,60,60,60,60,60)$ distribution of nonsynchronous trading probabilities, decreases by 24 percent from 0.2569 , for a portfolio of 500 , to 0.1954 for a portfolio of 20 securities. Whereas, for the $(24,29,44,80,80,80)$ distribution of nonsynchronous trading probabilities, it decreases by 27 percent from 0.3275 , for a portfolio of 500 securities to 0.2377 for a portfolio of 20 securities.

For any given distribution of non-trading probabilities, the magnitude of the fall in the value of the autocorrelation coefficient between large and small portfolios decreases as the heterogeneity of beta increases. For example, in the case of the $(24,29,44,80,80,80)$ distribution of 
nonsynchronous trading probabilities, the autocorrelation coefficient for the $(1,1,1,1,1,1)$ distribution of security betas reduces by 27 percent from 0.3150 in the case of a portfolio containing 500 securities to 0.2306 in the case of a portfolio containing 20 securities. Whereas, for the $(0.8,1.2,1.6,2.0,2.4,2.8)$ distribution of security betas, it decreases by 17 percent from 0.3679 in the case of a portfolio containing 500 securities, to 0.3050 in the case of a portfolio containing 20 securities. However, as was the case at the lower average nonsynchronous trading probabilities, this decrease appears to need both the mean and the variance of betas to have increased. For the $(0.8,0.9,1.0,1.0,1.1,1.2)$ distribution of security betas, the autocorrelation coefficient decreases by 27 percent, the same as in the homogeneous beta case.

In summary, the impact of heterogeneity of nonsynchronous trading probabilities and betas and the number of securities in the portfolio are broadly similar at different average levels of nonsynchronous trading. Having fewer securities in a portfolio reduces the autocorrelation coefficients, in a similar manner to what was observed in the homogeneous security cases. Heterogeneity in beta can act to offset this reduction, but only when the mean and the variance of the component securities have both increased above the values in the homogeneous case. Heterogeneity in nonsynchronous trading probability is relatively benign in its interaction with the number of securities in a portfolio, but may not offer as much offset, to the reduction in autocorrelation coefficient arising in portfolios of small numbers of securities, in situations of relatively high nonsynchronous trading probability.

\section{Conclusion}

In this study, we have examined the relationship between portfolio return autocorrelation and the number of securities in the portfolio, using hitherto concealed properties of the leading model of nonsynchronous trading, Lo and MacKinlay (1990). A key result of the original study is that there is a positive and strictly convex relation between stock trading infrequency and portfolio autocorrelation for portfolios where the number of securities has increased without limit. In this study, we demonstrate that for portfolios with a large, but finite, number of securities, a more complex relation exists between portfolio size and non-trading frequency that permits both zero and possibly large negative autocorrelation coefficients. Specifically, we identify an adjustment factor to the autocorrelations predicted by the Lo and MacKinlay (1990) model, which depends 
upon the means, variances and covariances of the component securities in the portfolio, and that is independent of the return measurement interval.

We show that at levels of nonsynchronous trading observed in well-developed markets, our adjustment factor implies that the return autocorrelations predicted by the Lo and MacKinlay (1990) model can be substantially lower than has been suggested previously, particularly at very short return intervals. However, we also show that while the likelihood of these lower predicted values increases with the probability of non-trading, it decreases with increased heterogeneity of the securities. This means that the subsequent predictions by Boudoukh et al (1994) are much less affected by the use of finite sized portfolios, at least for relatively modest levels of nonsynchronous trading. Our results for heterogeneous portfolios also reveal a rich structure of relationships between the number of securities in a portfolio, and the distributions of nonsynchronous trading probabilities and betas among those component securities. We find that the interaction of nonsynchronous trading probabilities and the number of component securities in a portfolio is relatively benign, except at relatively high probability levels, whereas the influence of beta heterogeneity can act to offset the reduction in autocorrelation coefficient arising from using finite sized portfolios. Overall, heterogeneity among the component securities tends to increase autocorrelation coefficients by more than the use of finite sized portfolios reduces them, except at very high level of security nonsynchronous trading, suggesting that making allowances for the heterogeneity of securities in the application of the Lo and MacKinlay (1990) model is essential to ensure it is most robust to applications involving portfolios of finite size.

For markets characterized by high levels of thin trading, our results have two further implications. First, models that adjust risk measures for portfolio autocorrelation by reference to the extent of thin trading may have the potential to over-correct the measures if the predicted autocorrelation is actually quite low. Conversely, one could fail to adjust for extreme levels of thin trading if one only examines the portfolio returns autocorrelation coefficients, since these can feasibly take low values when thin trading is extensive in the data. Second, the observation of both high and homogeneous levels of infrequent trading and high levels of portfolio return autocorrelation must mean that factors, other than nonsynchronous trading, are generating the observed autocorrelations. What these might be is beyond the scope of this study, but if trading is nonetheless highly infrequent, and in the absence of any structural weaknesses in a market, it may be very difficult to identify such economic or behavioural factors. 


\section{References}

Atchison, M.D., K.C. Butler, and R.R. Simonds, 1987, Nonsynchronous Security Trading and Market Index Autocorrelation, Journal of Finance, 42, 111-118.

Boulatov, A., T. Hendershott and D. Livdan, 2013, Informed trading and portfolio returns, Reviewof Economic Studies, 80, 35-72.

Boudoukh, J., M.P. Richardson, and R.F. Whitelaw, 1994, A Tale of Three Schools: Insights on Autocorrelation of Short-Horizon Stock Returns, Review of Financial Studies, 7, 539-

Brindranath, S., J. Kale and T. Noe, 1995, Of shepherds, sheep and the cross-autocorrelations in equity returns, Review of Financial Studies, 8, 401-430.

Chamberlain, G., 1983, Funds, factors, and diversification in arbitrage pricing models, Econometrica, 51, 1305-1332.

Chamberlain, G., and M. Rothschild, 1983, Arbitrage, factor structure, and mean variance analysis on large asset markets, Econometrica, 51, 1305-1332.

Conrad, J. and G. Kaul, 1988, Time Varying Expected Returns, Journal of Business, 61, 409-425.

Cohen, K., G. Hawawini, S. Maier, R. Schwartz and D. Whitcomb, 1983, Friction in the trading process and the estimation of systematic risk, Journal of Financial Economics, 12, 263-278.

Dimson, E., 1979, Risk Measurement when Shares are subject to Infrequent Trading, Journal of Financial Economics, 7, 197-226.

Dimson, E., P. Marsh and M. Staunton, 2001, Millenium Book II: 101 Years of Investment Returns,ABN AMRO and London Business School.

Fisher L., 1966, Some New Stock Market Indexes, Journal of Business, 39, 191-225.

Foerster S., and D. Keim, 1993, Non-trading, bid-ask spreads, and daily return autocorrelations, working paper, University of Pennsylvania.

Griffin, J., and R. Oomen, 2011, Covariance Measurement in the Presence of Non-Synchronous Trading and Market Microstructure Noise, Journal of Econometrics, 160,58-68.

Kadlec G.B., and D.M. Patterson, 1999, A transaction data analysis of nonsynchronous trading, Review of Financial Studies, 12, 609-630.

Keim D., 1989, Trading patterns, bid-ask spreads, and estimated security returns: The case of common stocks at calendar turning points, Journal of Financial Economics, 25, 75-98.

Keim D., and R.F. Stambaugh, 1986, Predicting returns in the stock and bond markets, Journal of Financial Economics, 17, 357-390.

Lesmond, D.A., J.P. Ogden and C.A. Trzcinka, 1999,ANew Estimate of Transaction Costs, Reviewof Financial Studies, 12, 1113-1141.

Lo A.W., and A.C. MacKinlay, 1990, An Econometric Analysis of Nonsynchronous Trading, Journal of Econometrics, 45, 181-211.

Martens, M., 2004, Estimating unbiased and precise realized covariances, unpublished working paper, Econometrics Institute, Erasmus University, The Netherlands.

Mech, T., 1993, Portfolio return autocorrelation, Journal of Financial Economics, 34, 307-344. 
Perry P.R., 1985, Portfolio Serial Correlation and Non-synchronous Trading, Journal of Financial and Quantitative Analysis, 20, 517-523.

Scholes M., and J. Williams, 1977, Estimating Betas from Nonsynchronous Data, Journal of Financial Economics, 5, 309-327.

Sias, R., and L. Stark, 1997, Return autocorrelation and institutional investors, Journal of FinancialEconomics, 46, 103-131.

Statman M., 1987, How Many Stocks Make a Diversified Portfolio?, Journal of Financial and Quantitative Analysis, 22, 353-63.

Steeley, J., 1997, The Nature and Implications of Serial Diversification, Advances in Investment Analysis and Portfolio Management, 4, 115-154.

Zhang, L., 2011, Estimating covariation: Epps effect, microstructure noise, Journal of Econometrics, 160, 33-47. 


\section{Appendix}

\section{Proof of Proposition 1}

The first-order autocorrelation of the observed returns, $R_{t}, t=1,2, \ldots, T$, for a portfolio of $i=$ $1,2, \ldots, n$, securities is

$$
\operatorname{Corr}\left(R_{t}, R_{t+1}\right)=\operatorname{Cov}\left(R_{t}, R_{t+1}\right) / \operatorname{Var}\left(R_{t}\right)
$$

where

$$
R_{t}=\sum_{i=1}^{n} \omega_{i} R_{i, t}, \quad \text { subject to } \sum_{i=1}^{n} \omega_{i}=1
$$

and where $R_{i, t}$ is the return on security $i$ at time $t$, and $\omega_{i}$ is the weight on security $i$ in the portfolio. For an equally-weighted portfolio, we obtain on substituting (A1.2) into (A1.1),

$$
\operatorname{Corr}\left(R_{t}, R_{t+1}\right)=\frac{\sum_{i=1}^{n} \operatorname{Cov}\left(R_{i, t}, R_{i, t+1}\right)+\sum_{i=1}^{n} \sum_{j=1, \neq i}^{n} \operatorname{Cov}\left(R_{i, t}, R_{j, t+1}\right)}{\sum_{i=1}^{n} \operatorname{Var}\left(R_{i, t}\right)+\sum_{i=1}^{n} \sum_{j=1, \neq i}^{n} \operatorname{Cov}\left(R_{i, t}, R_{j, t}\right)}
$$

which expresses the portfolio return autocorrelation in terms of the autocovariances, crossautocovariances, covariances and variances of the returns of the component securities.

Each of the components of the right hand side of (A1.3) can be expressed in terms of the following properties of the Lo and MacKinlay (1990) model, that is,

$$
\begin{array}{cl}
\operatorname{Cov}\left(R_{i, t}, R_{i, t+s}\right)= \begin{cases}\sigma_{i}^{2}+\frac{2 p_{i}}{\left(1-p_{i}\right)} \mu_{i}^{2} & \text { for } s=0 \\
-\mu_{i}^{2} p_{i} & \text { for } s=1\end{cases} \\
\operatorname{Cov}\left(R_{i, t}, R_{j, t+s}\right)= \begin{cases}\frac{\left(1-p_{i}\right)\left(1-p_{j}\right)}{\left(1-p_{i} p_{j}\right)} \beta_{i} \beta_{j} \sigma_{\Lambda}^{2} & \text { for } s=0 \\
\frac{\left(1-p_{i}\right)\left(1-p_{j}\right)}{\left(1-p_{i} p_{j}\right)} p_{j} \beta_{i} \beta_{j} \sigma_{\Lambda}^{2} & \text { for } s=1\end{cases}
\end{array}
$$

where $\beta_{i}$ is the loading for security $i$ on a zero-mean common factor, which has variance $\sigma_{\Lambda}^{2}, \mu_{i}$ and $\sigma_{i}^{2}$ are the expected return and variance of security $i$ and $p_{i}$ is the non-trading probability for security $i$. Substituting (A1.4) and (A1.5) into (A1.3) gives

$$
\operatorname{Corr}\left(R_{t}, R_{t+1}\right)=\frac{\sum_{i=1}^{n}\left(-\mu_{i}^{2} p_{i}\right)+\sum_{i=1}^{n} \sum_{j=1, \neq i}^{n} \frac{\left(1-p_{i}\right)\left(1-p_{j}\right)}{\left(1-p_{i} p_{j}\right)} p_{j} \beta_{i} \beta_{j} \sigma_{\Lambda}^{2}}{\sum_{i=1}^{n}\left(\sigma_{i}^{2}+\frac{2 p_{i}}{\left(1-p_{i}\right)} \mu_{i}^{2}\right)+\sum_{i=1}^{n} \sum_{j=1, \neq i}^{n} \frac{\left(1-p_{i}\right)\left(1-p_{j}\right)}{\left(1-p_{i} p_{j}\right)} \beta_{i} \beta_{j} \sigma_{\Lambda}^{2}}
$$


Making a representative security assumption, and setting $\beta_{i}=\beta_{i}=\beta, p_{i}=p_{j}=p, \mu_{i}=\mu$ and $\sigma_{i}=\sigma$ gives, after expanding the denominators of the covariance terms,

$$
\operatorname{Corr}\left(R_{t}, R_{t+1}\right)=\frac{\sum_{i=1}^{n}\left(-\mu^{2} p\right)+\sum_{i=1}^{n} \sum_{j=1, \neq i}^{n} \frac{(1-p)(1-p)}{(1-p)(1+p)} p \beta^{2} \sigma_{\Lambda}^{2}}{\sum_{i=1}^{n}\left(\sigma^{2}+\frac{2 p}{(1-p)} \mu^{2}\right)+\sum_{i=1}^{n} \sum_{j=1, \neq i}^{n} \frac{(1-p)(1-p)}{(1-p)(1+p)} \beta^{2} \sigma_{\Lambda}^{2}}
$$

Summing the component variance and covariance terms gives

$$
\operatorname{Corr}\left(R_{t}, R_{t+1}\right)=\frac{-n \mu^{2} p+n(n-1) \frac{(1-p)(1-p)}{(1-p)(1+p)} p \beta^{2} \sigma_{\Lambda}^{2}}{n \sigma^{2}+\frac{2 n p}{(1-p)} \mu^{2}+n(n-1) \frac{(1-p)(1-p)}{(1-p)(1+p)} \beta^{2} \sigma_{\Lambda}^{2}}
$$

which can be simplified by multiplying through by $(1+p)(1-p) / n$ to give

$$
\operatorname{Corr}\left(R_{t}, R_{t+1}\right)=\frac{-\mu^{2} p(1-p)(1+p)+(n-1)(1-p)(1-p) p \beta^{2} \sigma_{\Lambda}^{2}}{\sigma^{2}(1-p)(1+p)+2 p(1+p) \mu^{2}+n(n-1)(1-p)(1-p) \beta^{2} \sigma_{\Lambda}^{2}}
$$

Adding and subtracting $2 p(1-p)(n-1) \beta^{2} \sigma_{\Lambda}^{2}$ to the denominator gives

$$
\operatorname{Corr}\left(R_{t}, R_{t+1}\right)=\frac{-\mu^{2} p(1-p)(1+p)+(n-1)(1-p)(1-p) p \beta^{2} \sigma_{\Lambda}^{2}}{\sigma^{2}(1-p)(1+p)+2 p(1+p) \mu^{2}+(n-1)(1-p)(1+p) \beta^{2} \sigma_{\Lambda}^{2}-2 p(1-p)(n-1) \beta^{2} \sigma_{\Lambda}^{2}}
$$

and allows the simplification

$$
\operatorname{Corr}\left(R_{t}, R_{t+1}\right)=\frac{p(1-p)\left[(n-1)(1-p) \beta^{2} \sigma_{\Lambda}^{2}-\mu^{2}(1+p)\right]}{(1+p)\left[(n-1)(1-p) \beta^{2} \sigma_{\Lambda}^{2}+\sigma^{2}(1-p)\right]-2 p\left[(n-1)(1-p) \beta^{2} \sigma_{\Lambda}^{2}-\mu^{2}(1+p)\right]}
$$

which, using the common factor in the numerator and denominator, can be rearranged as

$$
\operatorname{Corr}\left(R_{t}, R_{t+1}\right)=\frac{p(1-p)}{(1+p)\left[\frac{(n-1)(1-p) \beta^{2} \sigma_{\Lambda}^{2}+\sigma^{2}(1-p)}{(n-1)(1-p) \beta^{2} \sigma_{\Lambda}^{2}-\mu^{2}(1+p)}\right]-2 p}
$$

Defining (as equation (8) in the text)

$$
\theta=\frac{(n-1)(p-1) \beta^{2} \sigma_{\Lambda}^{2}+\sigma^{2}(1-p)}{(n-1)(p-1) \beta^{2} \sigma_{\Lambda}^{2}-\mu^{2}(1+p)}
$$

gives equation (7) in the text,

$$
\operatorname{Corr}\left(R_{t}, R_{t+1}\right)=\frac{(1-p) p}{\theta(1+p)-2 p}
$$




\section{Proof of Proposition 2}

For portfolio returns aggregated over $q$ periods, where the aggregated periods are indexed by, $\tau$ so that one unit of $\tau$ time is equal to $q$ units of $t$ time, the first order autocorrelation coefficient is given by

$$
\operatorname{Corr}\left(R_{\tau}(q), R_{\tau+1}(q)\right)=\operatorname{Cov}\left(R_{\tau}(q), R_{\tau+1}(q)\right) / \operatorname{Var}\left(R_{\tau}(q)\right)
$$

where

$$
R_{\tau}(q)=\sum_{i=1}^{n} \omega_{i} R_{\mathrm{i}, \tau}(q), \quad \text { subject to } \sum_{i=1}^{n} \omega_{i}=1
$$

and where $R_{\mathrm{i}, \tau}(q)$ is the return on security $i$ at time $\tau$, where one unit of $\tau$ time is equal to $q$ units of $t$ time. For an equally-weighted portfolio, we obtain on substituting (A2.2) into (A2.1),

$$
\operatorname{Corr}\left(R_{\tau}(q), R_{\tau+1}(q)\right)=\frac{\sum_{i=1}^{n} \operatorname{Cov}\left(R_{i, \tau}(q), R_{i, \tau+1}(q)\right)+\sum_{i=1}^{n} \sum_{j=1, \neq i}^{n} \operatorname{Cov}\left(R_{i, \tau}(q), R_{j, \tau+1}(q)\right)}{\sum_{i=1}^{n} \operatorname{Var}\left(R_{i, \tau}(q)\right)+\sum_{i=1}^{n} \sum_{j=1, \neq i}^{n} \operatorname{Cov}\left(R_{i, \tau}(q), R_{j, \tau}(q)\right)}
$$

which expresses the time-aggregated portfolio return autocorrelation in terms of the timeaggregated autocovariances, cross-autocovariances, covariances and variances of the returns of the component securities.

Each of the components of the right hand side of (A2.3) can be expressed in terms of the following properties of the Lo and MacKinlay (1990) model, ${ }^{17}$ that is,

$$
\begin{array}{cc}
\operatorname{Cov}\left(R_{i, \tau}(q), R_{i, \tau+s}(q)\right)=\left\{\begin{array}{lr}
q \sigma_{i}^{2}+\frac{2 p_{i}\left(1-p_{i}^{q}\right)}{\left(1-p_{i}\right)^{2}} \mu_{i}^{2} & \text { for } s=0 \\
-\mu_{i}^{2} p_{i}\left(\frac{1-p_{i}^{q}}{1-p_{i}}\right)^{2} & \text { for } s=1
\end{array}\right. \\
\operatorname{Cov}\left(R_{i, \tau}(q), R_{j, \tau+s}(q)\right)= \begin{cases}{\left[q-\frac{p_{i}\left(1-p_{i}^{q}\right)\left(1-p_{j}\right)^{2}+p_{j}\left(1-p_{j}^{q}\right)\left(1-p_{i}\right)^{2}}{\left(1-p_{i}\right)\left(1-p_{j}\right)\left(1-p_{i} p_{j}\right)}\right.} \\
{\left[\begin{array}{ll}
\left(1-p_{i}\right)\left(1-p_{j}\right) \\
\left(1-p_{i} p_{j}\right)
\end{array}\left(\frac{1-p_{j}^{q}}{1-p_{j}}\right)^{2} \beta_{j} \beta_{\Lambda}^{2} \beta_{j} \sigma_{\Lambda}^{2}\right.} & \text { for } s=0\end{cases}
\end{array}
$$

where $\beta_{i}$ is the loading for security $i$ on a zero-mean common factor, which has variance $\sigma_{\Lambda}^{2}, \mu_{i}$ and $\sigma_{i}^{2}$ are the expected return and variance of security $i$ and $p_{i}$ is the non-trading probability for security $i$.

17. Equation (A2.5) uses the specification in Boudoukh et al (1994) equation (9), which corrects errors in the original specification in equation (3.6) of Lo and MacKinlay (1990). 
Making a representative security assumption, and setting $\beta_{i}=\beta_{i}=\beta, p_{i}=p_{j}=p, \mu_{i}=\mu$ and $\sigma_{i}=\sigma$ gives,

$$
\operatorname{Corr}\left(R_{\tau}(q), R_{\tau+1}(q)\right)=\frac{\sum_{i=1}^{n}-\mu^{2} p\left(\frac{1-p^{q}}{1-p}\right)^{2}+\sum_{i=1}^{n} \sum_{j=1, \neq i}^{n}(1-p)^{2}\left(\frac{1-p^{q}}{1-p}\right)^{2} p \beta^{2} \sigma_{\Lambda}^{2}}{\sum_{i=1}^{n} q \sigma^{2}+\frac{2 p\left(1-p^{q}\right)}{(1-p)^{2}} \mu^{2}+\sum_{i=1}^{n} \sum_{j=1, \neq i}^{n}\left[q-\frac{p(1-p)^{2}\left(1-p^{q}\right)+p(1-p)^{2}\left(1-p^{q}\right)}{(1-p)^{2}\left(1-p^{2}\right)}\right] \beta^{2} \sigma_{\Lambda}^{2}}
$$

which on summing over $n$ securities gives

$$
\operatorname{Corr}\left(R_{\tau}(q), R_{\tau+1}(q)\right)=\frac{-n \mu^{2} p\left(\frac{1-p^{q}}{1-p}\right)^{2}+n(n-1) \frac{(1-p)^{2}}{\left(1-p^{2}\right)}\left(\frac{1-p^{q}}{1-p}\right)^{2} p \beta^{2} \sigma_{\Lambda}^{2}}{n\left(q \sigma^{2}+\frac{2 p\left(1-p^{q}\right)}{(1-p)^{2}} \mu^{2}\right)+n(n-1)\left[q-\frac{p(1-p)^{2}\left(1-p^{q}\right)+p(1-p)^{2}\left(1-p^{q}\right)}{(1-p)^{2}\left(1-p^{2}\right)}\right] \beta^{2} \sigma_{\Lambda}^{2}}
$$

Cancelling the common factor $(1-p)^{2}$ in the covariance terms gives

$$
\operatorname{Corr}\left(R_{\tau}(q), R_{\tau+1}(q)\right)=\frac{-n \mu^{2} p\left(\frac{1-p^{q}}{1-p}\right)^{2}+n(n-1) \frac{\left(1-p^{q}\right)^{2}}{\left(1-p^{2}\right)} p \beta^{2} \sigma_{\Lambda}^{2}}{n\left(q \sigma^{2}+\frac{2 p\left(1-p^{q}\right)}{(1-p)^{2}} \mu^{2}\right)+n(n-1)\left[q-\frac{2 p\left(1-p^{q}\right)}{\left(1-p^{2}\right)}\right] \beta^{2} \sigma_{\Lambda}^{2}}
$$

Using a common divisor in the terms in the denominator of (A2.8) and expanding terms in all the divisors gives

$$
\operatorname{Corr}\left(R_{\tau}(q), R_{\tau+1}(q)\right)=\frac{\frac{-n \mu^{2} p\left(1-p^{q}\right)^{2}}{(1-p)(1-p)}+n(n-1) \frac{\left(1-p^{q}\right)^{2}}{(1-p)(1+p)} p \beta^{2} \sigma_{\Lambda}^{2}}{n\left(\frac{q \sigma^{2}(1-p)^{2}+2 p\left(1-p^{q}\right) \mu^{2}}{(1-p)(1-p)}\right)+n(n-1)\left[\frac{q\left(1-p^{2}\right)-2 p\left(1-p^{q}\right)}{(1-p)(1+p)}\right] \beta^{2} \sigma_{\Lambda}^{2}}
$$

Multiplying through by $(1-p)^{2}(1+p) / n$ gives

$$
\operatorname{Corr}\left(R_{\tau}(q), R_{\tau+1}(q)\right)=\frac{-\mu^{2} p\left(1-p^{q}\right)^{2}(1+p)+(n-1)\left(1-p^{q}\right)^{2}(1-p) p \beta^{2} \sigma_{\Lambda}^{2}}{\left(q \sigma^{2}(1-p)^{2}+2 p\left(1-p^{q}\right) \mu^{2}\right)(1+p)+(n-1)(1-p)\left[q\left(1-p^{2}\right)-2 p\left(1-p^{q}\right)\right] \beta^{2} \sigma_{\Lambda}^{2}}
$$

Rearranging terms gives

$$
\operatorname{Corr}\left(R_{\mathrm{\tau}}(q), R_{\tau+1}(q)\right)=\frac{p\left(1-p^{q}\right)^{2}\left[(n-1)(1-p) \beta^{2} \sigma_{\Lambda}^{2}-\mu^{2}(1+p)\right]}{q\left(1-p^{2}\right)\left[(n-1)(1-p) \beta^{2} \sigma_{\Lambda}^{2}+\sigma^{2}(1-p)\right]-2 p\left(1-p^{q}\right)\left[(n-1)(1-p) \beta^{2} \sigma_{\Lambda}^{2}-\mu^{2}(1+p)\right]}
$$

Defining (as equation (8) in the text)

$$
\theta=\frac{(n-1)(p-1) \beta^{2} \sigma_{\Lambda}^{2}+\sigma^{2}(1-p)}{(n-1)(p-1) \beta^{2} \sigma_{\Lambda}^{2}-\mu^{2}(1+p)}
$$

gives

$$
\operatorname{Corr}\left(R_{\tau}(q), R_{\tau+1}(q)\right)=\frac{\left(1-p^{q}\right)^{2} p}{\theta q\left(1-p^{2}\right)-2 p\left(1-p^{q}\right)}
$$

which is equation (11) in the text. 
Table 1: Estimated Portfolio Return Autocorrelations

\begin{tabular}{|c|c|c|c|c|c|c|c|c|c|c|}
\hline \multicolumn{11}{|c|}{$\begin{array}{l}\text { This table contains the autocorrelation coefficients for temporally aggregated portfolio returns, estimated using equations } \\
\text { (11) and (14). The portfolios are comprised of } n \text { securities having a common non-trading probability, } p \text {, a common reward } \\
\text { ratio, } \xi \text {, a common ratio of representative security return variance to common factor variance, } \phi \text {, and unit beta. }\end{array}$} \\
\hline \multirow{2}{*}{$n$} & \multirow{2}{*}{$p$} & \multicolumn{3}{|c|}{$\phi=2$} & \multicolumn{3}{|c|}{$\phi=4$} & \multicolumn{3}{|c|}{$\phi=9$} \\
\hline & & $\xi=0.19$ & $\xi=0.41$ & $\xi=0.65$ & $\xi=0.19$ & $\xi=0.41$ & $\xi=0.65$ & $\xi=0.19$ & $\xi=0.41$ & $\xi=0.65$ \\
\hline \multicolumn{11}{|c|}{ Panel A: Weekly returns } \\
\hline$n \rightarrow \infty$ & 27 & 0.0890 & 0.0890 & 0.0890 & 0.0890 & 0.0890 & 0.0890 & 0.0890 & 0.0890 & 0.0890 \\
\hline 500 & 27 & 0.0886 & 0.0886 & 0.0886 & 0.0882 & 0.0882 & 0.0882 & 0.0872 & 0.0872 & 0.0872 \\
\hline 100 & 27 & 0.0870 & 0.0870 & 0.0870 & 0.0850 & 0.0850 & 0.0850 & 0.0804 & 0.0804 & 0.0804 \\
\hline 20 & 27 & 0.0792 & 0.0792 & 0.0792 & 0.0713 & 0.0713 & 0.0713 & 0.0571 & 0.0571 & 0.0571 \\
\hline 10 & 27 & 0.0705 & 0.0705 & 0.0705 & 0.0584 & 0.0584 & 0.0584 & 0.0409 & 0.0408 & 0.0408 \\
\hline$n \rightarrow \infty$ & 60 & 0.2601 & 0.2601 & 0.2601 & 0.2601 & 0.2601 & 0.2601 & 0.2601 & 0.2601 & 0.2601 \\
\hline 500 & 60 & 0.2585 & 0.2585 & 0.2585 & 0.2569 & 0.2569 & 0.2569 & 0.2530 & 0.2529 & 0.2529 \\
\hline 100 & 60 & 0.2521 & 0.2521 & 0.2521 & 0.2446 & 0.2446 & 0.2445 & 0.2277 & 0.2276 & 0.2275 \\
\hline 20 & 60 & 0.2233 & 0.2232 & 0.2231 & 0.1956 & 0.1955 & 0.1953 & 0.1494 & 0.1492 & 0.1488 \\
\hline 10 & 60 & 0.1930 & 0.1929 & 0.1926 & 0.1534 & 0.1532 & 0.1528 & 0.1014 & 0.1011 & 0.1006 \\
\hline$n \rightarrow \infty$ & 80 & 0.5094 & 0.5094 & 0.5094 & 0.5094 & 0.5094 & 0.5094 & 0.5094 & 0.5094 & 0.5094 \\
\hline 500 & 80 & 0.5043 & 0.5043 & 0.5043 & 0.4993 & 0.4993 & 0.4992 & 0.4873 & 0.4872 & 0.4870 \\
\hline 100 & 80 & 0.4848 & 0.4846 & 0.4844 & 0.4624 & 0.4622 & 0.4618 & 0.4145 & 0.4141 & 0.4133 \\
\hline 20 & 80 & 0.4026 & 0.4022 & 0.4014 & 0.3329 & 0.3322 & 0.3309 & 0.2322 & 0.2313 & 0.2296 \\
\hline 10 & 80 & 0.3266 & 0.3259 & 0.3246 & 0.2403 & 0.2394 & 0.2378 & 0.1446 & 0.1437 & 0.1418 \\
\hline$n \rightarrow \infty$ & 95 & 0.8458 & 0.8458 & 0.8458 & 0.8458 & 0.8458 & 0.8458 & 0.8458 & 0.8458 & 0.8458 \\
\hline 500 & 95 & 0.8179 & 0.8173 & 0.8163 & 0.7917 & 0.7907 & 0.7887 & 0.7331 & 0.7311 & 0.7272 \\
\hline 100 & 95 & 0.7215 & 0.7193 & 0.7151 & 0.6291 & 0.6257 & 0.6192 & 0.4764 & 0.4718 & 0.4631 \\
\hline 20 & 95 & 0.4456 & 0.4409 & 0.4320 & 0.3024 & 0.2976 & 0.2888 & 0.1675 & 0.1635 & 0.1561 \\
\hline 10 & 95 & 0.2920 & 0.2872 & 0.2784 & 0.1763 & 0.1722 & 0.1646 & 0.0883 & 0.0851 & 0.0792 \\
\hline \multicolumn{11}{|c|}{ Panel B: Monthly returns } \\
\hline$n \rightarrow \infty$ & 27 & 0.0178 & 0.0178 & 0.0178 & 0.0178 & 0.0178 & 0.0178 & 0.0178 & 0.0178 & 0.0178 \\
\hline 500 & 27 & 0.0178 & 0.0178 & 0.0178 & 0.0177 & 0.0177 & 0.0177 & 0.0175 & 0.0175 & 0.0175 \\
\hline 100 & 27 & 0.0175 & 0.0175 & 0.0175 & 0.0171 & 0.0171 & 0.0171 & 0.0163 & 0.0163 & 0.0163 \\
\hline 20 & 27 & 0.0161 & 0.0161 & 0.0161 & 0.0146 & 0.0146 & 0.0146 & 0.0120 & 0.0120 & 0.0120 \\
\hline 10 & 27 & 0.0145 & 0.0145 & 0.0145 & 0.0122 & 0.0122 & 0.0122 & 0.0088 & 0.0088 & 0.0088 \\
\hline$n \rightarrow \infty$ & 60 & 0.0488 & 0.0488 & 0.0488 & 0.0488 & 0.0488 & 0.0488 & 0.0488 & 0.0488 & 0.0488 \\
\hline 500 & 60 & 0.0486 & 0.0486 & 0.0486 & 0.0483 & 0.0483 & 0.0483 & 0.0478 & 0.0478 & 0.0478 \\
\hline 100 & 60 & 0.0477 & 0.0477 & 0.0477 & 0.0467 & 0.0467 & 0.0467 & 0.0443 & 0.0443 & 0.0443 \\
\hline 20 & 60 & 0.0437 & 0.0437 & 0.0437 & 0.0396 & 0.0396 & 0.0396 & 0.0321 & 0.0320 & 0.0320 \\
\hline 10 & 60 & 0.0392 & 0.0392 & 0.0391 & 0.0328 & 0.0327 & 0.0327 & 0.0232 & 0.0232 & 0.0231 \\
\hline$n \rightarrow \infty$ & 80 & 0.1258 & 0.1258 & 0.1258 & 0.1258 & 0.1258 & 0.1258 & 0.1258 & 0.1258 & 0.1258 \\
\hline 500 & 80 & 0.1251 & 0.1251 & 0.1251 & 0.1245 & 0.1245 & 0.1245 & 0.1230 & 0.1230 & 0.1229 \\
\hline 100 & 80 & 0.1226 & 0.1226 & 0.1226 & 0.1197 & 0.1197 & 0.1196 & 0.1129 & 0.1128 & 0.1127 \\
\hline 20 & 80 & 0.1111 & 0.1110 & 0.1109 & 0.0995 & 0.0993 & 0.0991 & 0.0788 & 0.0786 & 0.0782 \\
\hline 10 & 80 & 0.0983 & 0.0982 & 0.0980 & 0.0807 & 0.0805 & 0.0801 & 0.0557 & 0.0554 & 0.0549 \\
\hline$n \rightarrow \infty$ & 95 & 0.5062 & 0.5062 & 0.5062 & 0.5062 & 0.5062 & 0.5062 & 0.5062 & 0.5062 & 0.5062 \\
\hline 500 & 95 & 0.5012 & 0.5011 & 0.5009 & 0.4962 & 0.4960 & 0.4956 & 0.4843 & 0.4838 & 0.4830 \\
\hline 100 & 95 & 0.4818 & 0.4813 & 0.4803 & 0.4596 & 0.4587 & 0.4569 & 0.4121 & 0.4104 & 0.4071 \\
\hline 20 & 95 & 0.4003 & 0.3984 & 0.3948 & 0.3310 & 0.3282 & 0.3228 & 0.2309 & 0.2271 & 0.2200 \\
\hline 10 & 95 & 0.3248 & 0.3219 & 0.3163 & 0.2389 & 0.2352 & 0.2282 & 0.1436 & 0.1394 & 0.1315 \\
\hline
\end{tabular}


Table 1 cont.

This table contains the autocorrelation coefficients for temporally aggregated portfolio returns, estimated using equations (11) and (14). The portfolios are comprised of $n$ securities having a common non-trading probability, $p$, a common reward ratio, $\xi$, a common ratio of representative security return variance to common factor variance, $\phi$, and unit beta.

\begin{tabular}{|c|c|c|c|c|c|c|c|c|c|c|}
\hline \multirow{2}{*}{$N$} & \multirow{2}{*}{$p$} & \multicolumn{3}{|c|}{$\phi=2$} & \multicolumn{3}{|c|}{$\phi=4$} & \multicolumn{3}{|c|}{$\phi=9$} \\
\hline & & $\xi=0.19$ & $\xi=0.41$ & $\xi=0.65$ & $\xi=0.19$ & $\xi=0.41$ & $\xi=0.65$ & $\xi=0.19$ & $\xi=0.41$ & $\xi=0.65$ \\
\hline \multicolumn{11}{|c|}{ Panel C: Quarterly returns } \\
\hline$n \rightarrow \infty$ & 27 & 0.0058 & 0.0058 & 0.0058 & 0.0058 & 0.0058 & 0.0058 & 0.0058 & 0.0058 & 0.0058 \\
\hline 500 & 27 & 0.0058 & 0.0058 & 0.0058 & 0.0058 & 0.0058 & 0.0058 & 0.0057 & 0.0057 & 0.0057 \\
\hline 100 & 27 & 0.0057 & 0.0057 & 0.0057 & 0.0056 & 0.0056 & 0.0056 & 0.0053 & 0.0053 & 0.0053 \\
\hline 20 & 27 & 0.0052 & 0.0052 & 0.0052 & 0.0048 & 0.0048 & 0.0048 & 0.0039 & 0.0039 & 0.0039 \\
\hline 10 & 27 & 0.0047 & 0.0047 & 0.0047 & 0.0040 & 0.0040 & 0.0040 & 0.0029 & 0.0029 & 0.0029 \\
\hline$n \rightarrow \infty$ & 60 & 0.0153 & 0.0153 & 0.0153 & 0.0153 & 0.0153 & 0.0153 & 0.0153 & 0.0153 & 0.0153 \\
\hline 500 & 60 & 0.0152 & 0.0152 & 0.0152 & 0.0151 & 0.0151 & 0.0151 & 0.0150 & 0.0150 & 0.0150 \\
\hline 100 & 60 & 0.0150 & 0.0150 & 0.0150 & 0.0147 & 0.0147 & 0.0147 & 0.0140 & 0.0140 & 0.0140 \\
\hline 20 & 60 & 0.0138 & 0.0138 & 0.0138 & 0.0125 & 0.0125 & 0.0125 & 0.0103 & 0.0102 & 0.0102 \\
\hline 10 & 60 & 0.0124 & 0.0124 & 0.0124 & 0.0105 & 0.0105 & 0.0104 & 0.0075 & 0.0075 & 0.0075 \\
\hline$n \rightarrow \infty$ & 80 & 0.0364 & 0.0364 & 0.0364 & 0.0364 & 0.0364 & 0.0364 & 0.0364 & 0.0364 & 0.0364 \\
\hline 500 & 80 & 0.0363 & 0.0363 & 0.0363 & 0.0361 & 0.0361 & 0.0361 & 0.0357 & 0.0357 & 0.0357 \\
\hline 100 & 80 & 0.0356 & 0.0356 & 0.0356 & 0.0349 & 0.0349 & 0.0349 & 0.0332 & 0.0332 & 0.0331 \\
\hline 20 & 80 & 0.0327 & 0.0327 & 0.0327 & 0.0297 & 0.0297 & 0.0296 & 0.0241 & 0.0240 & 0.0240 \\
\hline 10 & 80 & 0.0294 & 0.0294 & 0.0293 & 0.0246 & 0.0246 & 0.0245 & 0.0175 & 0.0175 & 0.0173 \\
\hline$n \rightarrow \infty$ & 95 & 0.1929 & 0.1929 & 0.1929 & 0.1929 & 0.1929 & 0.1929 & 0.1929 & 0.1929 & 0.1929 \\
\hline 500 & 95 & 0.1918 & 0.1918 & 0.1918 & 0.1908 & 0.1907 & 0.1906 & 0.1881 & 0.1880 & 0.1879 \\
\hline 100 & 95 & 0.1876 & 0.1875 & 0.1873 & 0.1825 & 0.1823 & 0.1819 & 0.1710 & 0.1706 & 0.1698 \\
\hline 20 & 95 & 0.1680 & 0.1675 & 0.1666 & 0.1488 & 0.1480 & 0.1463 & 0.1156 & 0.1142 & 0.1116 \\
\hline 10 & 95 & 0.1469 & 0.1460 & 0.1444 & 0.1186 & 0.1172 & 0.1146 & 0.0799 & 0.0780 & 0.0743 \\
\hline \multicolumn{11}{|c|}{ Panel D: Annual returns } \\
\hline$n \rightarrow \infty$ & 27 & 0.0016 & 0.0016 & 0.0016 & 0.0016 & 0.0016 & 0.0016 & 0.0016 & 0.0016 & 0.0016 \\
\hline 500 & 27 & 0.0016 & 0.0016 & 0.0016 & 0.0015 & 0.0015 & 0.0015 & 0.0015 & 0.0015 & 0.0015 \\
\hline 100 & 27 & 0.0015 & 0.0015 & 0.0015 & 0.0015 & 0.0015 & 0.0015 & 0.0014 & 0.0014 & 0.0014 \\
\hline 20 & 27 & 0.0014 & 0.0014 & 0.0014 & 0.0013 & 0.0013 & 0.0013 & 0.0011 & 0.0011 & 0.0011 \\
\hline 10 & 27 & 0.0013 & 0.0013 & 0.0013 & 0.0011 & 0.0011 & 0.0011 & 0.0008 & 0.0008 & 0.0008 \\
\hline$n \rightarrow \infty$ & 60 & 0.0040 & 0.0040 & 0.0040 & 0.0040 & 0.0040 & 0.0040 & 0.0040 & 0.0040 & 0.0040 \\
\hline 500 & 60 & 0.0040 & 0.0040 & 0.0040 & 0.0040 & 0.0040 & 0.0040 & 0.0040 & 0.0040 & 0.0040 \\
\hline 100 & 60 & 0.0040 & 0.0040 & 0.0040 & 0.0039 & 0.0039 & 0.0039 & 0.0037 & 0.0037 & 0.0037 \\
\hline 20 & 60 & 0.0037 & 0.0036 & 0.0036 & 0.0033 & 0.0033 & 0.0033 & 0.0027 & 0.0027 & 0.0027 \\
\hline 10 & 60 & 0.0033 & 0.0033 & 0.0033 & 0.0028 & 0.0028 & 0.0028 & 0.0020 & 0.0020 & 0.0020 \\
\hline$n \rightarrow \infty$ & 80 & 0.0094 & 0.0094 & 0.0094 & 0.0094 & 0.0094 & 0.0094 & 0.0094 & 0.0094 & 0.0094 \\
\hline 500 & 80 & 0.0093 & 0.0093 & 0.0093 & 0.0093 & 0.0093 & 0.0093 & 0.0092 & 0.0092 & 0.0092 \\
\hline 100 & 80 & 0.0092 & 0.0092 & 0.0092 & 0.0090 & 0.0090 & 0.0090 & 0.0086 & 0.0086 & 0.0086 \\
\hline 20 & 80 & 0.0084 & 0.0084 & 0.0084 & 0.0077 & 0.0077 & 0.0077 & 0.0063 & 0.0063 & 0.0063 \\
\hline 10 & 80 & 0.0076 & 0.0076 & 0.0076 & 0.0064 & 0.0064 & 0.0064 & 0.0046 & 0.0046 & 0.0046 \\
\hline$n \rightarrow \infty$ & 95 & 0.0434 & 0.0434 & 0.0434 & 0.0434 & 0.0434 & 0.0434 & 0.0434 & 0.0434 & 0.0434 \\
\hline 500 & 95 & 0.0432 & 0.0432 & 0.0432 & 0.0430 & 0.0430 & 0.0430 & 0.0426 & 0.0426 & 0.0425 \\
\hline 100 & 95 & 0.0425 & 0.0425 & 0.0424 & 0.0416 & 0.0415 & 0.0415 & 0.0395 & 0.0394 & 0.0392 \\
\hline 20 & 95 & 0.0389 & 0.0388 & 0.0387 & 0.0353 & 0.0351 & 0.0348 & 0.0286 & 0.0283 & 0.0277 \\
\hline 10 & 95 & 0.0349 & 0.0344 & 0.0344 & 0.0292 & 0.0289 & 0.0284 & 0.0207 & 0.0202 & 0.0194 \\
\hline
\end{tabular}


Table 2: Estimated Autocorrelations for returns to heterogeneous portfolios

\begin{tabular}{|c|c|c|c|c|c|c|c|c|c|c|}
\hline \multicolumn{11}{|c|}{$\begin{array}{l}\text { This table contains the weekly return autocorrelation coefficients, estimated from equations (11) and (14), for portfolios } \\
\text { comprised of } 6 \text { groups of securities, classified by beta }(., ., ., ., ., \text { and non-trading probability }[., ., ., ., ., .] \text {, which comprise the } \\
\text { fractions } 5,20,25,25,20 \text { and } 5 \text { percent of the portfolio. Size refers to the number of securities in the portfolio; } \\
\text { autocorrelations alongside the blank size cells are the asymptotic values. } \xi \text { is the common reward ratio for firms in the } \\
\text { portfolio. }\end{array}$} \\
\hline \multirow{3}{*}{$\begin{array}{l}\text { Non-trading } \\
\text { probability }\end{array}$} & \multirow{3}{*}{ Size } & \multicolumn{9}{|c|}{ Betas } \\
\hline & & \multicolumn{3}{|c|}{$(1,1,1,1,1,1)$} & \multicolumn{3}{|c|}{$(.8, .9,1,1,1.1,1.2)$} & \multicolumn{3}{|c|}{$(.8,1 \cdot 2,1 \cdot 6,2 \cdot 0,2 \cdot 4,2 \cdot 8)$} \\
\hline & & $\xi=0.19$ & $\xi=0.41$ & $\xi=0.65$ & $\xi=0.19$ & $\xi=0.41$ & $\xi=0.65$ & $\xi=0.19$ & $\xi=0.41$ & $\xi=0.65$ \\
\hline \multicolumn{11}{|c|}{ (a) Low average non-trading probability } \\
\hline \multirow[t]{4}{*}[27,27,27,27,27,27]{} & $n \rightarrow \infty$ & 0.0890 & 0.0890 & 0.0890 & 0.0890 & 0.0890 & 0.0890 & 0.0890 & 0.0890 & 0.0890 \\
\hline & 500 & 0.0882 & 0.0882 & 0.0882 & 0.0882 & 0.0882 & 0.0882 & 0.0886 & 0.0886 & 0.0886 \\
\hline & 100 & 0.0850 & 0.0850 & 0.0850 & 0.0850 & 0.0850 & 0.0850 & 0.0870 & 0.0870 & 0.0869 \\
\hline & 20 & 0.0713 & 0.0713 & 0.0713 & 0.0713 & 0.0713 & 0.0713 & 0.0791 & 0.0791 & 0.0791 \\
\hline \multirow[t]{4}{*}[0,11,21,32,44,55]{} & $n \rightarrow \infty$ & 0.1033 & 0.1033 & 0.1033 & 0.1072 & 0.1072 & 0.1072 & 0.1160 & 0.1160 & 0.1160 \\
\hline & 500 & 0.1023 & 0.1023 & 0.1023 & 0.1062 & 0.1062 & 0.1062 & 0.1155 & 0.1155 & 0.1155 \\
\hline & 100 & 0.0986 & 0.0986 & 0.0986 & 0.1023 & 0.1023 & 0.1023 & 0.1132 & 0.1132 & 0.1132 \\
\hline & 20 & 0.0828 & 0.0828 & 0.0827 & 0.0856 & 0.0856 & 0.0856 & 0.1025 & 0.1024 & 0.1024 \\
\hline \multirow[t]{4}{*}[0,0,11,32,60,85]{} & $n \rightarrow \infty$ & 0.1318 & 0.1318 & 0.1318 & 0.1420 & 0.1420 & 0.1420 & 0.1643 & 0.1643 & 0.1643 \\
\hline & 500 & 0.1305 & 0.1305 & 0.1305 & 0.1405 & 0.1405 & 0.1405 & 0.1634 & 0.1634 & 0.1634 \\
\hline & 100 & 0.1256 & 0.1256 & 0.1256 & 0.1350 & 0.1350 & 0.1350 & 0.1600 & 0.1600 & 0.1600 \\
\hline & 20 & 0.1050 & 0.1050 & 0.1050 & 0.1121 & 0.1121 & 0.1120 & 0.1439 & 0.1439 & 0.1438 \\
\hline \multirow[t]{4}{*}[0,0,0,43,60,85]{} & $n \rightarrow \infty$ & 0.1397 & 0.1397 & 0.1397 & 0.1508 & 0.1508 & 0.1508 & 0.1782 & 0.1782 & 0.1782 \\
\hline & 500 & 0.1383 & 0.1383 & 0.1383 & 0.1493 & 0.1493 & 0.1493 & 0.1772 & 0.1772 & 0.1772 \\
\hline & 100 & 0.1331 & 0.1331 & 0.1331 & 0.1435 & 0.1435 & 0.1434 & 0.1735 & 0.1735 & 0.1734 \\
\hline & 20 & 0.1115 & 0.1114 & 0.1113 & 0.1193 & 0.1193 & 0.1192 & 0.1562 & 0.1562 & 0.1561 \\
\hline \multicolumn{11}{|c|}{ (b) High average non-trading probability } \\
\hline \multirow[t]{4}{*}[60,60,60,60,60,60]{} & $n \rightarrow \infty$ & 0.2601 & 0.2601 & 0.2601 & 0.2601 & 0.2601 & 0.2601 & 0.2601 & 0.2601 & 0.2601 \\
\hline & 500 & 0.2569 & 0.2569 & 0.2569 & 0.2569 & 0.2569 & 0.2569 & 0.2585 & 0.2585 & 0.2585 \\
\hline & 100 & 0.2446 & 0.2446 & 0.2445 & 0.2446 & 0.2446 & 0.2445 & 0.2521 & 0.2521 & 0.2521 \\
\hline & 20 & 0.1956 & 0.1955 & 0.1953 & 0.1955 & 0.1954 & 0.1952 & 0.2231 & 0.2230 & 0.2229 \\
\hline \multirow[t]{4}{*}[39,47,56,65,72,80]{} & $n \rightarrow \infty$ & 0.2775 & 0.2775 & 0.2775 & 0.2855 & 0.2855 & 0.2855 & 0.3044 & 0.3044 & 0.3044 \\
\hline & 500 & 0.2740 & 0.2740 & 0.2740 & 0.2817 & 0.2817 & 0.2817 & 0.3023 & 0.3023 & 0.3023 \\
\hline & 100 & 0.2604 & 0.2604 & 0.2603 & 0.2675 & 0.2675 & 0.2674 & 0.2940 & 0.2940 & 0.2940 \\
\hline & 20 & 0.2067 & 0.2065 & 0.2063 & 0.2114 & 0.2113 & 0.2109 & 0.2569 & 0.2568 & 0.2566 \\
\hline \multirow[t]{4}{*}[24,39,51,73,80,80]{} & $n \rightarrow \infty$ & 0.3059 & 0.3059 & 0.3059 & 0.3179 & 0.3179 & 0.3179 & 0.3499 & 0.3499 & 0.3499 \\
\hline & 500 & 0.3017 & 0.3017 & 0.3017 & 0.3133 & 0.3133 & 0.3133 & 0.3472 & 0.3472 & 0.3472 \\
\hline & 100 & 0.2857 & 0.2857 & 0.2855 & 0.2963 & 0.2962 & 0.2961 & 0.3367 & 0.3367 & 0.3366 \\
\hline & 20 & 0.2237 & 0.2235 & 0.2231 & 0.2304 & 0.2302 & 0.2298 & 0.2905 & 0.2903 & 0.2900 \\
\hline \multirow[t]{4}{*}[24,39,44,80,80,80]{} & $n \rightarrow \infty$ & 0.3197 & 0.3197 & 0.3197 & 0.3325 & 0.3325 & 0.3325 & 0.3709 & 0.3709 & 0.3709 \\
\hline & 500 & 0.3150 & 0.3150 & 0.3150 & 0.3275 & 0.3275 & 0.3275 & 0.3679 & 0.3679 & 0.3679 \\
\hline & 100 & 0.2976 & 0.2976 & 0.2974 & 0.3089 & 0.3088 & 0.3087 & 0.3562 & 0.3562 & 0.3561 \\
\hline & 20 & 0.2309 & 0.2306 & 0.2301 & 0.2380 & 0.2377 & 0.2372 & 0.3052 & 0.3050 & 0.3046 \\
\hline
\end{tabular}


Figure 1

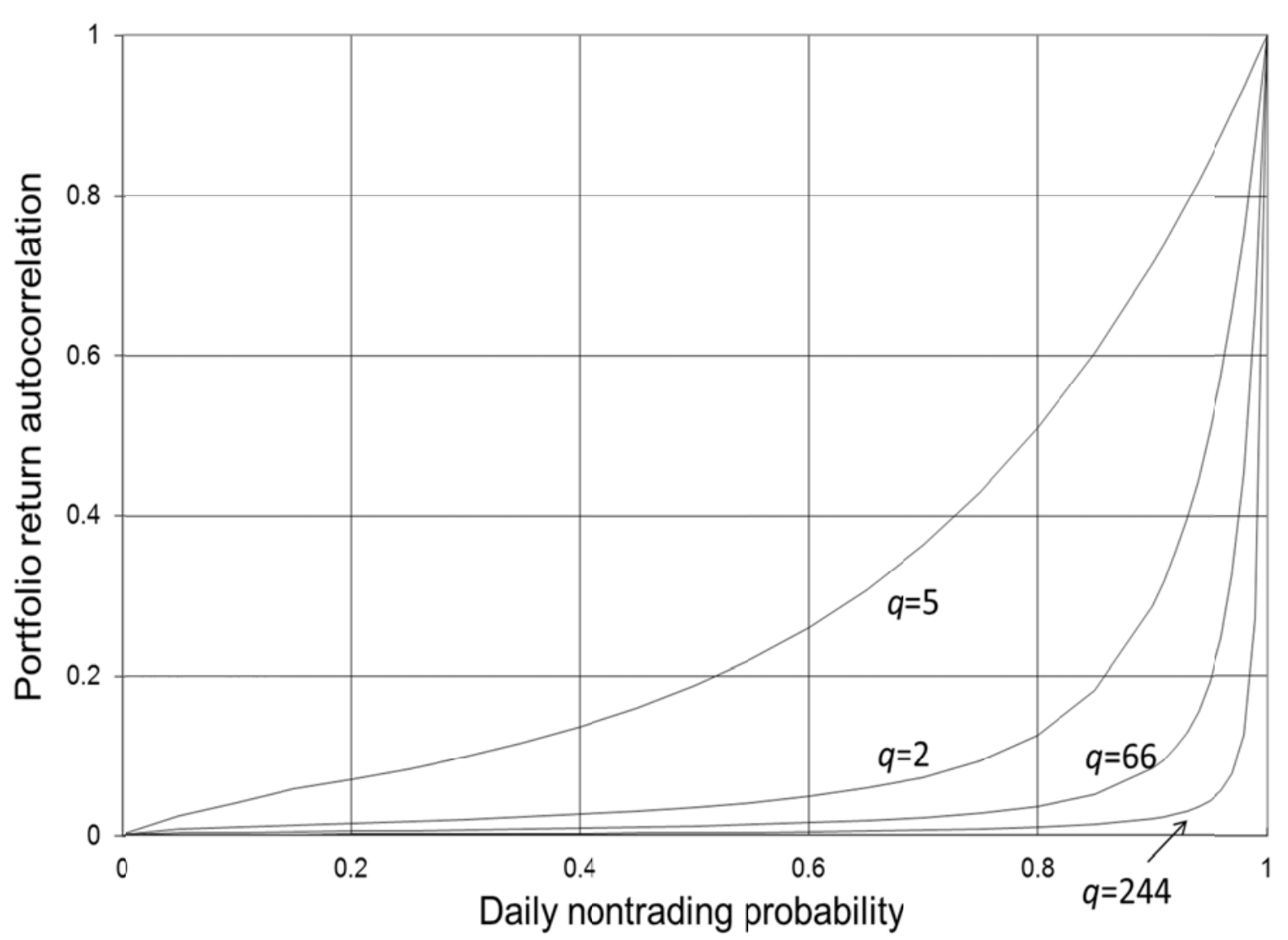

First order autocorrelation of temporally aggregated portfolio returns as a function of per period (daily) non-trading probability, where $q$ is the aggregation value, which is measured in days. 
Figure 2

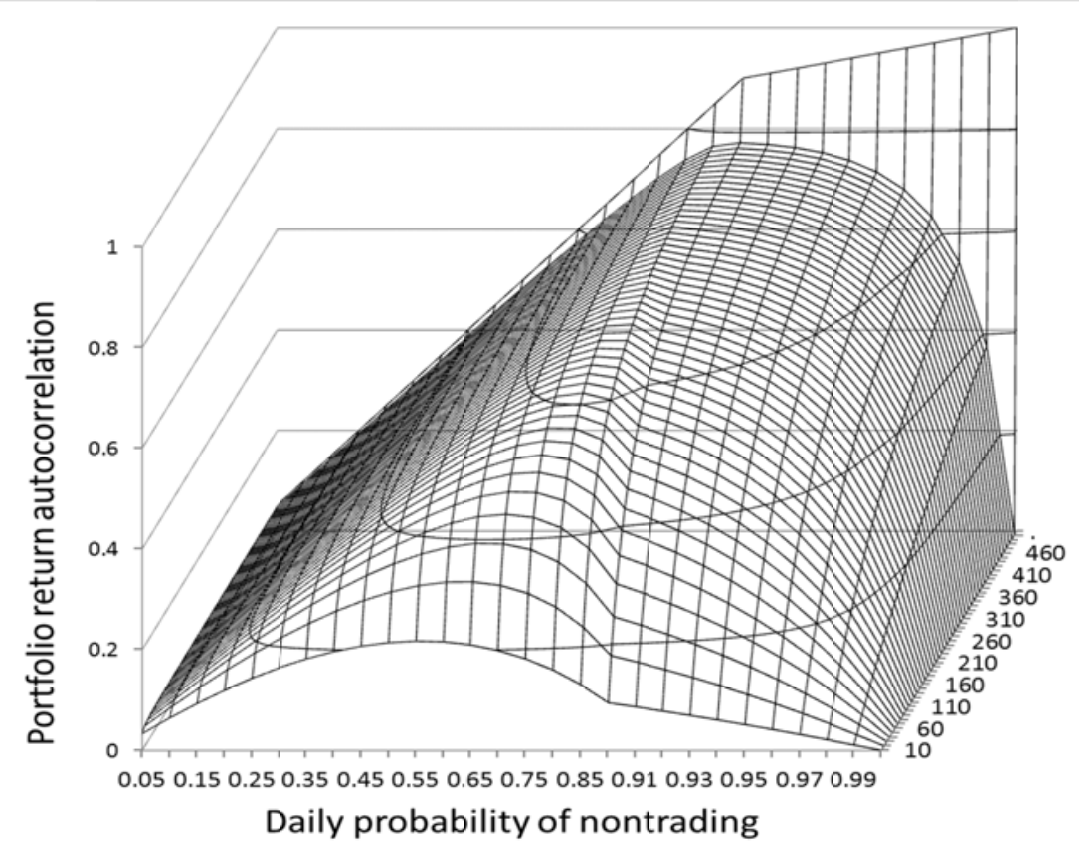

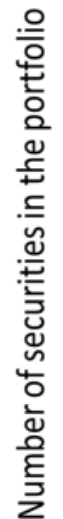

(a) Daily returns

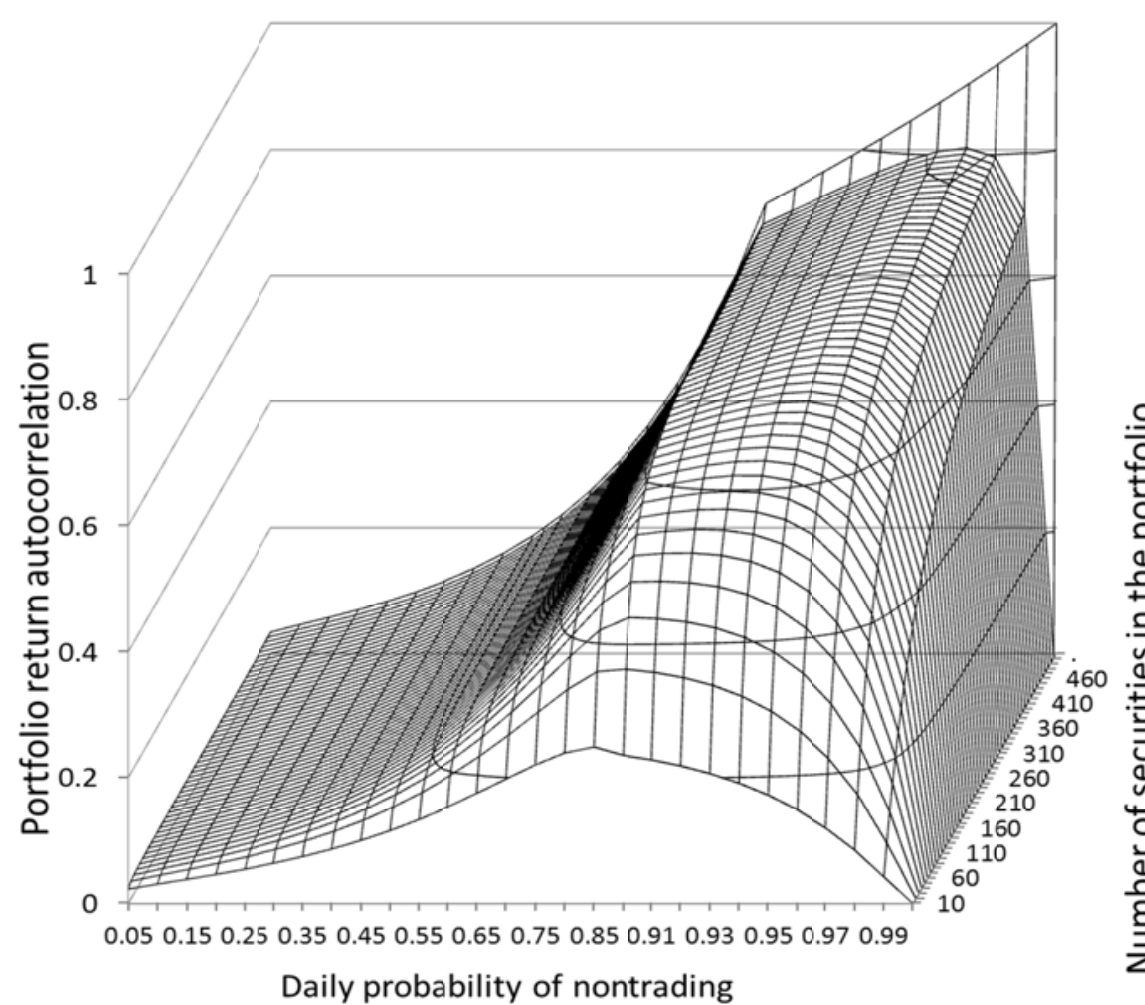

(b) Weekly returns 


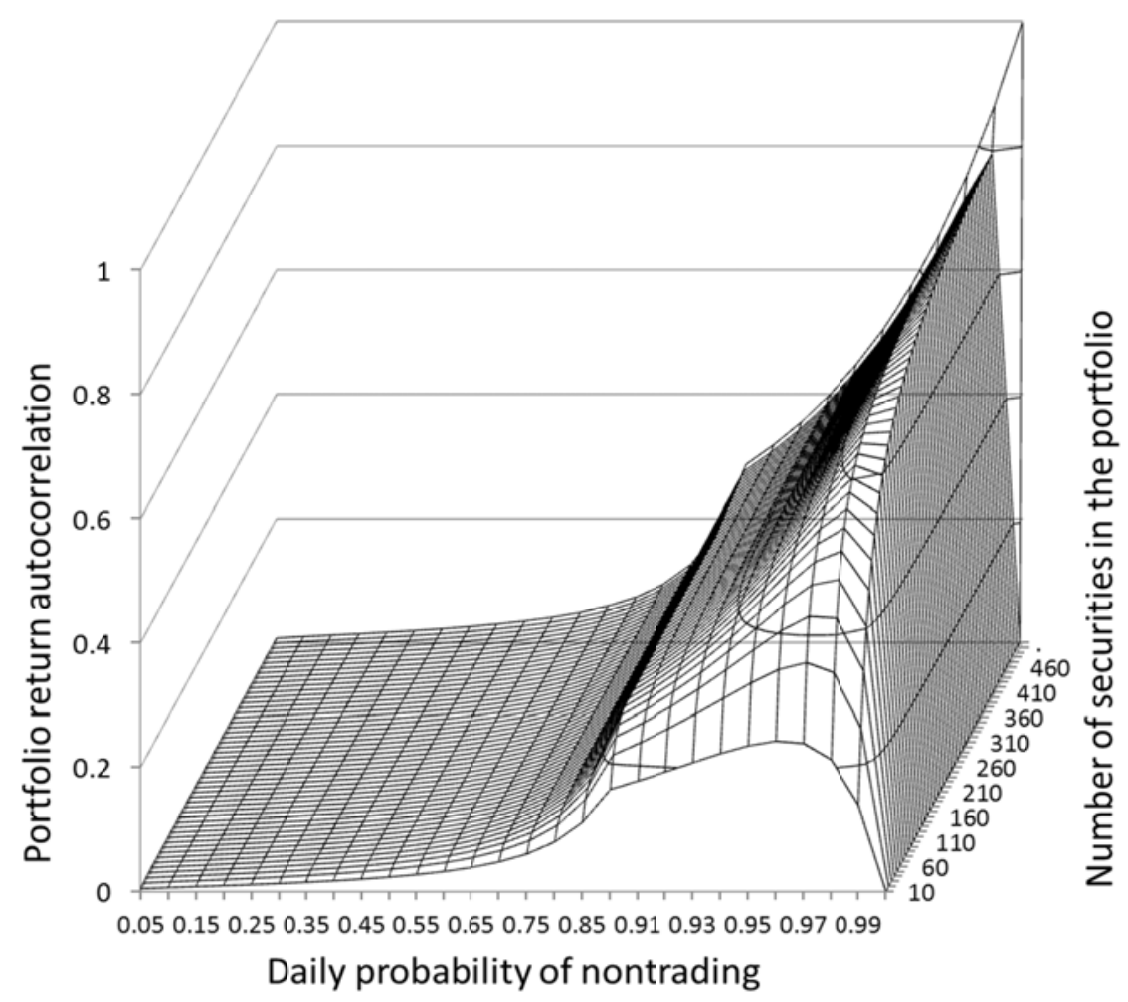

(c) Monthly returns

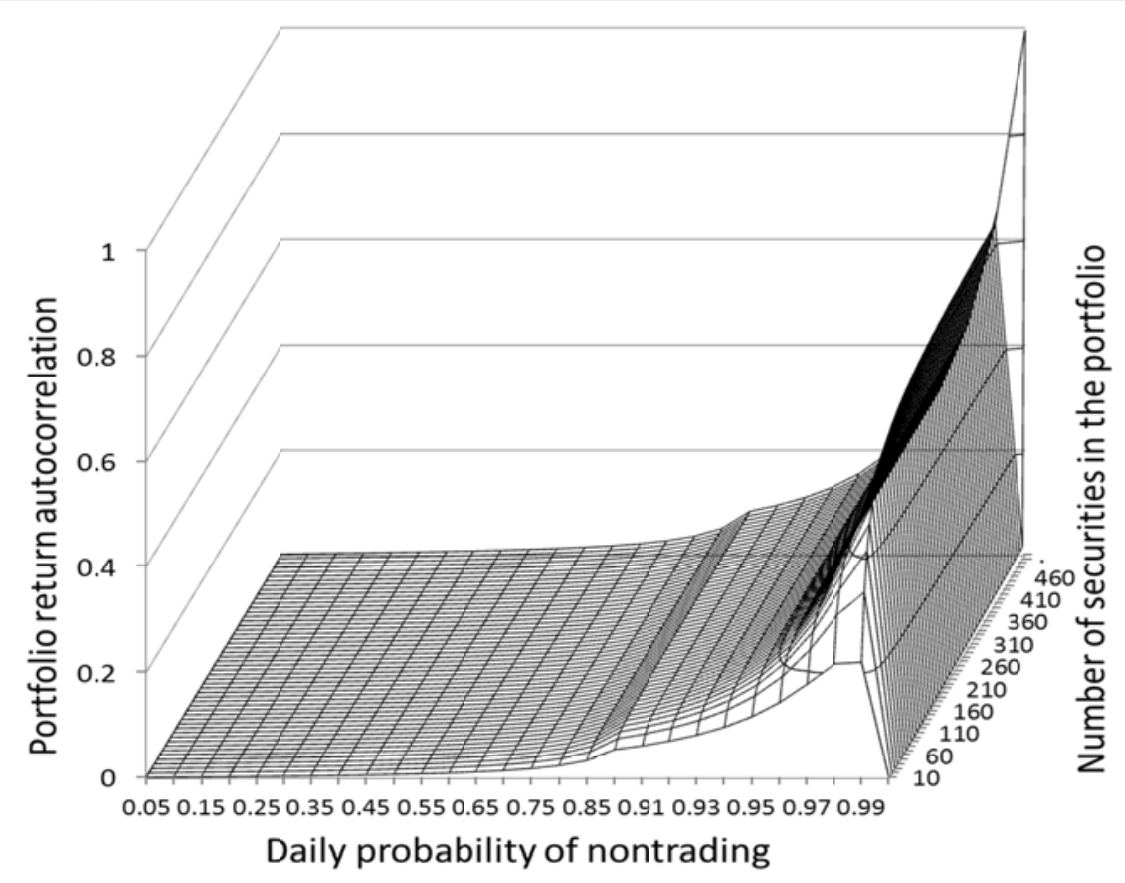

(d) Quarterly returns 


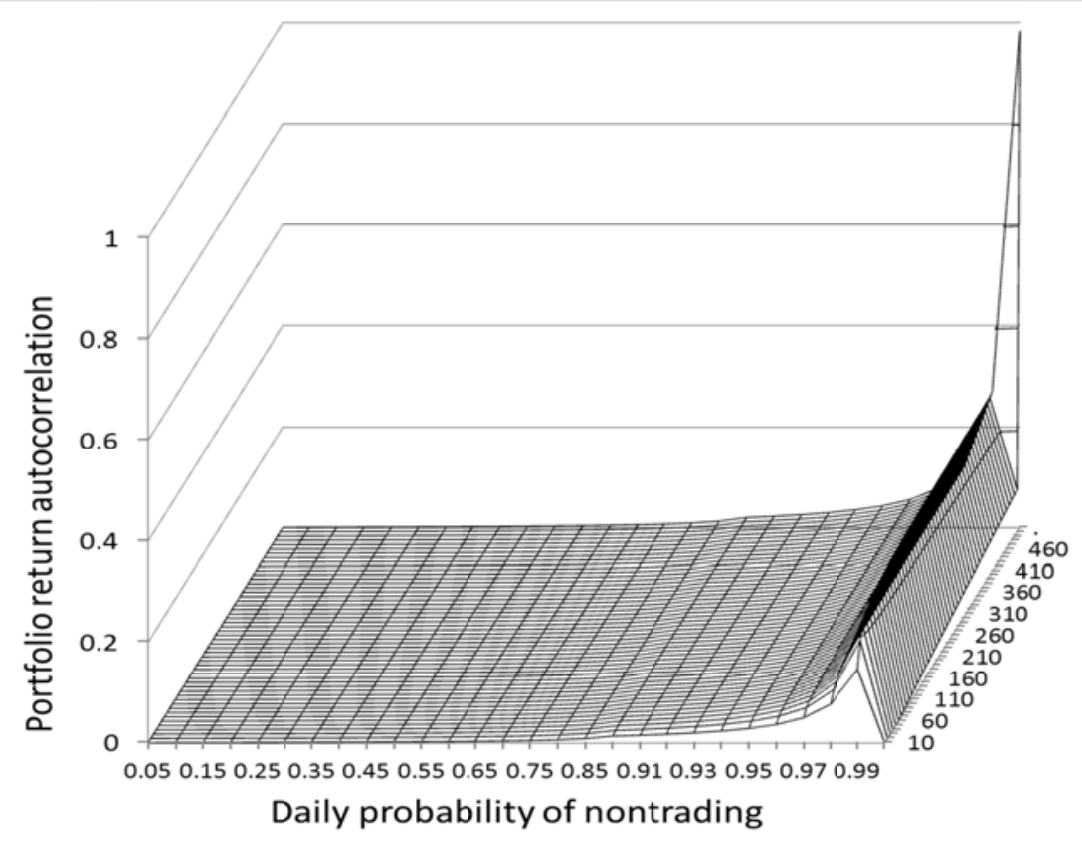

(e) Annual returns

First order autocorrelation of portfolio returns as a function of the daily non-trading probability and portfolio size. Securities in the portfolios have equal non-trading probabilities, unit betas, total risk equal to double the risk of the common factor and reward ratio of 0.41 . Autocorrelations for asymptotically large portfolios are plotted at the rear of each graph. The horizontal scale changes at $p=0.90$ to better display the downward slope. 
Figure 3

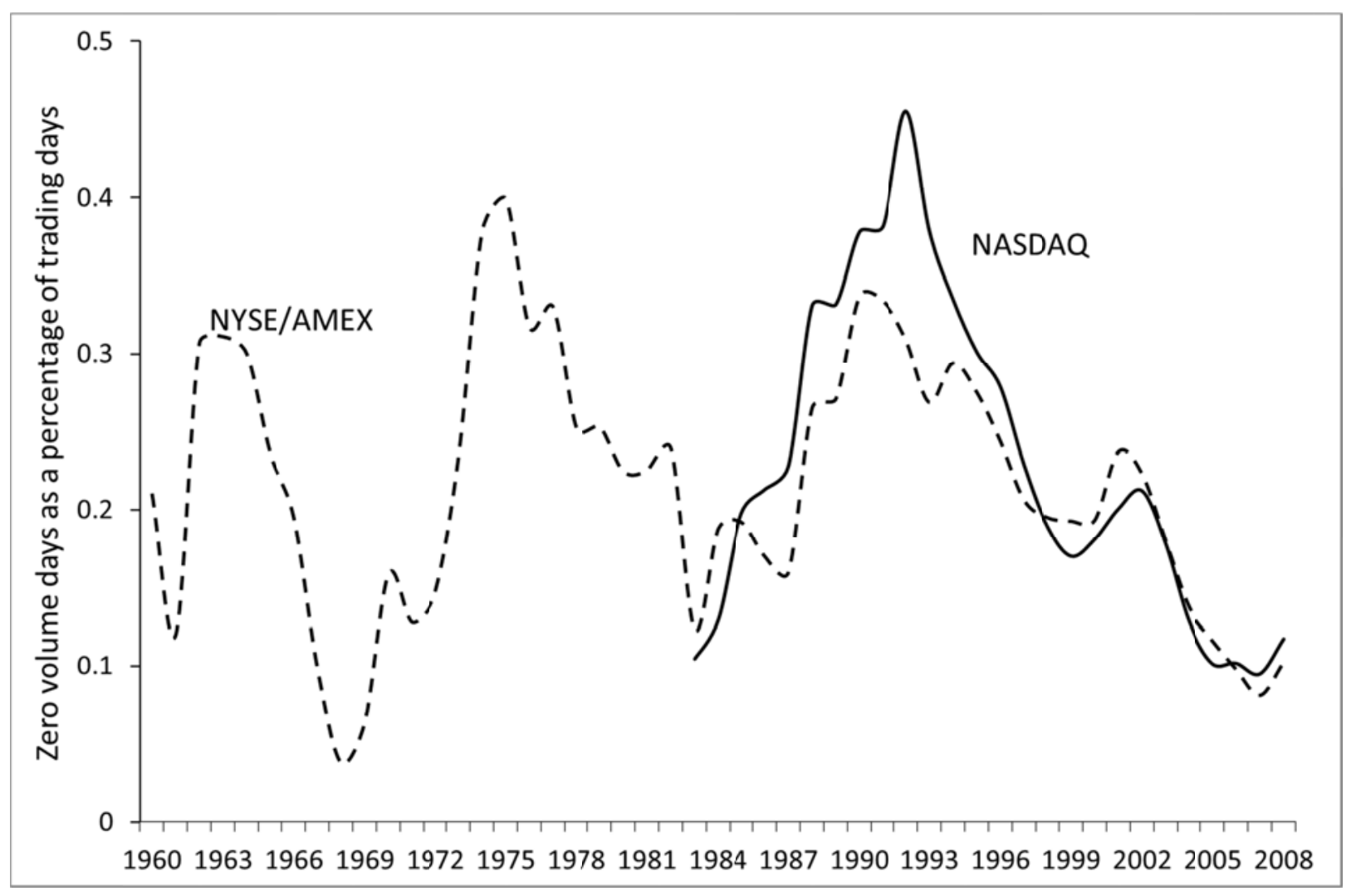

The average, across all stocks in the smallest capitalization decile for a given year, of the number of zero volume days as a proportion of total trading days for a stock. The sample period is 19602008 for NYSE/AMEX firms and 1983-2008 for NASDAQ firms. 